\title{
A rapidly Mixing Monte Carlo Method for the Simulation of Slow Molecular Processes
}

\author{
V. Durmaz, K. Fackeldey, M. Weber \\ Zuse Institute Berlin
}

Germany

\section{Introduction}

Since the middle of the last century, the continously increasing computational power has been adopted to molecular modeling and the simulation of molecular dynamics as well. In this field of research, one is interested in the dynamical behaviour of molecular systems. In contrast to the beginnings when only single or very few atoms could be simulated, the systems under consideration have grown to the size of macromolecules like proteins, DNA, or membrane structures nowadays resulting in high-dimensional conformational spaces. This development is triggered by permanently increasing computational power, the utilization of massively parallel hardware as well as improved algorithms and enhanced molecular force fields, covering chemical and especially biological molecular systems at a progressive rate. Applications basing on molecular modeling help to understand and predict molecular phenomena in various fields of applications providing information on e. $\mathrm{g}$. molecular conformations and recognition, protein folding, drug-design, or binding affinities. Typical fields benefiting from their usage are pharmacy, medicine, chemistry and materials research.

Unfortunately, often the atomistic structure is so complex that a satisfactory mapping of the processes can hardly be realized, due to the large number of atoms and in particular, the difference in time scales. More precisely, for the molecular function of a protein for example, its folding is a key issue. In contrast to this folding event that may last up to several seconds or even minutes, the time step of an ordinary trajectory based molecular simulation is linked to the fastest molecular oscillation which occurs in case of the chemical $H-C$ bond with a time period around few $10^{-15}$ seconds. Even today, exorbitant computational effort and time need to be invested in order to capture such interesting processes.

\section{Atomistic simulations}

The molecular simulation methods can be divided into two classes: the deterministic and the stochastic approaches. The first one is also known as the classical molecular dynamcis (MD), which relies on classical mechanics as described by Newton's Equations of motion (e.g. Frenkel \& Smit (1996); Griebel et al. (2007)). The latter class is known as Monte Carlo Methods (e.g. Binder \& Landau (2000)). Both methods have celebrated a great success in various applications. For an overview, we refer to Leach (2001) and Schlick (2002). 
In the forthcoming we will briefly introdcue the molecular dynamics and then give a more detailed introdcution into the Monte Carlo method within its framework. Later on, we will eploit the commonalities between both simulation methods in order to explain the hybrid Monte Carlo method.

\subsection{Deterministic molecular simulations}

The basic idea of molecular dynamics is to calculate trajectories containing spatial coordinates of atoms evolved in time. Let us assume, a state $x=(q, p) \in \mathbb{R}^{6 n}$ of a molecular system with $n$ atoms is given in a 6n-dimensional phase space. Here, $q \in \Omega \subset \mathbb{R}^{3 n}$ and $p \in \Gamma \subset \mathbb{R}^{3 n}$ denote the position coordinates in the position space $\Omega$ and the momentum cooridnates in the momentum space $\Gamma$, respectively. For a classical description of molecular motion with conservative forces, Newton's second law is given by the equation

$$
M \ddot{q}=f(q),
$$

where $M \in \mathbb{R}^{3 n \times 3 n}$ is a diagonal matrix of atomic masses, $f(q) \in \mathbb{R}^{3 n}$ is a vector of internal and external forces acting on the atoms at position $q$, and $\ddot{q}$ stands for the acceleration as the second time derivative of $q$. The total energy of the system under consideration, i.e. the Hamiltonian $H$ is given by

$$
H(p, q)=V(q)+K(p),
$$

where $K(p)=\frac{1}{2} p^{T} M^{-1} p$ and $V(q)$ represent the kinetic and the poteintial part, respectively. Since the kinetic part only depends on the momenta and the potential part on the positions only, the Hamiltonian is separable, leading to the following equations of motion:

$$
\begin{aligned}
& \dot{q}=\frac{\partial H(p, q)}{\partial p}=\frac{\partial K(p)}{\partial p}=M^{-1} p \\
& \dot{p}=\frac{\partial H(p, q)}{\partial q}=\frac{\partial V(q)}{\partial q}=-\nabla_{q} V(q) .
\end{aligned}
$$

Inserting these two time derivatives $\dot{q}$ and $\dot{p}$ into the time derivative of the Hamiltonian

$$
\frac{\mathrm{d} H(p, q)}{\mathrm{d} t}=\underbrace{\left(\nabla_{q} H\right)}_{=-\dot{p}} \dot{q}+\underbrace{\left(\nabla_{p} H\right)}_{=\dot{q}} \dot{p}=-\dot{p} \dot{q}+\dot{p} \dot{q}=0
$$

shows that the total energy according to Hamilton is constant over time, i. e. $H(q(t), q(t))=$ $H(q(0), q(0))$ for $t \in(-\infty, \infty)$. Time discretization during simulation is achieved by applying a constant step size integrator like the Sörmer-Verlet (Verlet (1967)) or leap-frog (Hockney (1970)) integrator for which the time step size $\Delta t$ is determined by the shortest oscillation period of bonds in a molecule, namely some femtoseconds. In addition, a time integration for a sampling scheme as represented by Equation (2) has to fulfil certain properties like reversibility and symplecticity. Besides the fact that the time step is confined to a small size, the total time span $\tau$, i.e. the time of the simulation, has to be chosen carefully as well. More precisely, for a given initial state $x(0)$ and a slightly perturbated state $x^{*}(0)$ it can be shown:

$$
\left\|x(t)-x^{*}(t)\right\| \sim\left\|x(0)-x^{*}(0)\right\| \exp \left(\lambda_{\max } t\right)
$$

which means that the error $\left\|x(t)-x^{*}(t)\right\|$ depends exponentially on the time, where $\lambda_{\max }$ is the maximal Lyapunov characteristic exponent. It has been shown by Deuflhard et al. 
(1999), that for molecular dynamics $\lambda_{\max }$ may be very large such that only trajectories of some $10^{-13}$ seconds are correlated with the initial state. Due to the large $\lambda_{\max }$ value, molecular dynamics is chaotic. However, if one is interested in the probability of rare events during this chaotic process, e. g. protein folding, the deterministic trajectory based approach seems not favourable. Here, we do not focus on these aspects and refer to Leimkuhler \& Reich (2004) for details.

Having briefly scetched the idea of the classical molecular dynamics, we now change our point of view to the concept of statistical dynamics and investigate ensembles of molecular systems.

\subsection{Statistical molecular simulations}

In statistical molecular dynamcis, we are not interested in the movement or speed of each particle but in averaged macroscopic properties of the atoms. The advantage of this approach lies in the fact that many physical properties in which we are interested, like energies, enthalpies and entropies do not depend strongly on a detailed dynamical movement of each particle but on a collection of particles. In other words, we are no longer interested in each particle but in a system of particles and its probability to be in a certain state. In order to describe this state, we need the term "ensemble" which is an imaginary collection of systems described by the same Hamiltonian where each system is in a unique mircoscopic state at any given instant time.

In an $(n, V, T)$-ensemble, each subsystem, in our example of section 4 it is a single molecule in vacuum, has the same macroscopic properties volume $V$ and temperature T. Furthermore, neither chemical reactions take place nor do particles escape which means that the number $n$ of atoms is also kept constant. The subsystems can only exchange energy with their surroundings and therefore, have different states $x=(q, p)$ to which Boltzmann statistics can be applied. For a detailed derivation of the Boltzmann distribution which is defined as the most probable distribution of states in an $(n, V, T)$-ensemble and is also called canonical ensemble $\mu_{c a n}$, see Schäfer (1960), pp. 5-11. Via the Hamiltonian $H(\cdot)$, the probability that a molecule attains the state $x$ is

$$
\mu_{\text {can }}(x)=\frac{1}{Z} \exp (-\beta H(x)),
$$

where $Z$ is a corresponding normalization. $\beta$ is the inverse temperature

$$
\beta=\frac{1}{k_{B} T},
$$

where $T$ is measured in Kelvin and $k_{B} \approx 1.38066 \cdot 10^{-23} \mathrm{JK}^{-1}$ is the Boltzmann constant.

\subsubsection{Partition functions}

For separable Hamiltonian functions, the Boltzmann distribution of molecular states $x=$ $(q, p)$ in the canonical ensemble $\mu_{c a n}(x)=\pi(q) \eta(p)$ has the following splitting into a distribution of momenta and position states:

$$
\eta(p)=\frac{1}{Z_{p}} \exp (-\beta K(p)), \quad \pi(q)=\frac{1}{Z_{q}} \exp (-\beta V(q)) .
$$

$\eta(\cdot)$ represents a Gaussian distribution for each of the $3 n$ momenta coordinates, because $K$ is a quadratic function with the diagonal matrix $M$. 
Therefore, creating a sequence of momenta numerically (sampling) according to their Boltzmann distribution $\eta(p)$ is simple, see e.g. Allen \& Tildesley (1987). The spacial factor $\pi(\cdot)$ is a more complex distribution function. Whereas the exponential function $\exp (-\beta V(q))$ can be computed pointwise, the normalization constant (also denoted as spatial partition function ${ }^{1}$ )

$$
Z_{q}=\int_{\Omega} \exp (-\beta V(q)) \mathrm{d} q
$$

is unknown. For a sampling of points $q \in \Omega$ according to a distribution which is known except for a normalization constant, the Metropolis-Hastings algorithm can be applied, see section 3 .

\subsubsection{Bracket notation}

A macroscopic measurement is always carried out for a snapshot of a molecular ensemble, where molecular states are distributed according to the Boltzmann distribution. A spatial observable $\langle A\rangle_{\pi}$ of a function $A: \Omega \rightarrow \mathbb{R}$ in configuration space, e. g. potential energies or torsion angles, is therefore measured as an ensemble mean, i. e. an expectation value

$$
\langle A\rangle_{\pi}=\int_{\Omega} A(q) \pi(q) \mathrm{d} q
$$

where $A$ is $\mu$-Lebesgue integrable w. r. t. the measure $\mu(\mathrm{d} q):=\pi(q) \mathrm{d} q$. In the following, these bracket notations for observable and inner products will be often used abbreviations.

\subsubsection{Importance sampling}

In Equation 5 the dimension of the space $\Omega$ is proportional to the number of degrees of freedom. The evaluation of this integral leads to a high-dimensional numerical integration problem. Thus, a regular or equidistant grid in the phase space combined with a standard deterministic integration scheme, such as Simpsons rule cannot be used (see Table 1).

\begin{tabular}{|c|c|}
\hline Method & Convergene \\
\hline \hline Trapeziodal & $N^{-2 / d}$ \\
\hline Simpson & $N^{-4 / d}$ \\
\hline Monte Carlo & $N^{-1 / 2}$ \\
\hline
\end{tabular}

Table 1. The convergence behaviour of the determinstic quadrature rules (Simpson and Trapezoidal) depend on the dimension: With increasing number of dimension, the convergence rate growth. In contrast, the convergence behavior of the Monte Carlo method does not depend on the dimension

In a Monte Carlo method one solves numerically

$$
\int_{\Omega} g(x) \mathrm{d} x \approx \frac{1}{N} \sum_{i=1}^{N} g\left(x_{i}\right) .
$$

The key issue in a Monte Carlo method, is that the evaluation points $x_{1}, \ldots, x_{N}$ are statistically selected. There are many ways how to distribute the points. If they are distributed uniformly, it is called a simple sampling.

\footnotetext{
${ }^{1} Z_{q}$ is a function of temperature, volume and number of particles of an ensemble. For the canonical ensemble $Z_{q}$ is a constant. The total partition function $Z=Z_{q} Z_{p}$ is the key to calculating all macroscopic properties of the system.
} 
However, for complex functions like $\pi(q)$, a simple sampling is inappropriate since the uniform distribution of particles might lead to an insufficient reproduction of the function (see center distribution of Fig. 1). Fig. 1 illustrates well that it is not easy to generate a good distribution of particles in a reasonable amount of computer time. In the importance sampling, the integrand is modified in order to yield an expectation of a quantity that varies less than the original integrand over the region of integration. In order to solve Equation (5), one applies Monte Carlo integration methods with an importance sampling routine, i.e.

$$
\langle A\rangle_{\pi} \approx \frac{1}{N} \sum_{i=1}^{N} A\left(q_{i}\right), \quad q_{i} \propto \pi,
$$

where $N$ position states $q_{i} \in \Omega$ are sampled according to their Boltzmann distribution $\pi$. Hence, the expectation value of an observable can be simply approximated by its arithmetic mean. For introductory literature of Monte Carlo integration methods see Hammersley \& Handscomb (1964) and Robert \& Casella (1999).

Since the major part of the conformational space $\Omega$ is physically irrelevant due to high potential energies $V(q)$, only few conformations have a probability substantially larger than zero. Instead of creating independent points $q_{i}$, in practice, one starts with a physically relevant position state $q_{1} \in \Omega$ and generates further states via a Markov chain

$$
q_{1} \rightarrow q_{2} \rightarrow \ldots \rightarrow q_{N}
$$

Summing up, both approaches have advantages and disadvantages. In classical molecular dynamics simulations the motion of each individual particle can be described deterministically. However, long simulations are hardly feasible. On the other hand, the statistical molecular simulation is capable of handling large molecular systems but lacks of a trajectory.

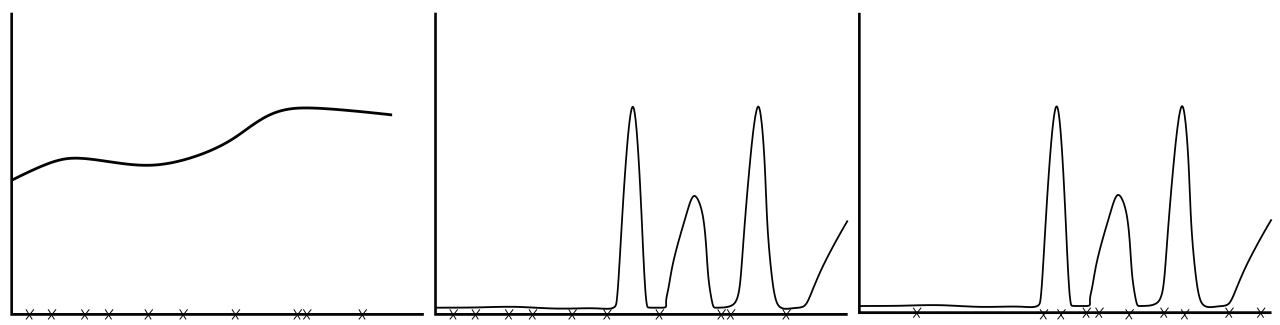

Fig. 1. Left: A simple sampling with a uniformly distributed set of particles fitting a nearly constant function. Center: A simple sampling inappropriate for unconstant distribution functions. Right: A suitable distribution of points well fitting the desired function (importance sampling).

\section{Hybrid Monte Carlo method (HMC)}

The conclusion of the forgoing section is a motivation to construct a method which profits from either of the two approaches. This has been done in the hybrid Monte Carlo method. Roughly speaking, this method can be seen as a combination of deterministic molecular dynamics combined with stochastic impulses. So far we have elucidated the basic framework of statistical simulations but not their numerical realization in detail. This will be done in the next prargraphs. 


\subsection{Detailed balance criterion}

A sufficient condition for a correct sampling via Monte Carlo Simulation in the situation of Equation 7 is the detailed balance condition with the desired Boltzmann distribution $\pi$. This condition holds, if the conditional probability density function $\mathbf{P}(q \rightarrow \widetilde{q})$ for a transition $q \rightarrow \widetilde{q}$ in Equation 7 meets

$$
\pi(q) \mathbf{P}(q \rightarrow \widetilde{q})=\pi(\widetilde{q}) \mathbf{P}(\widetilde{q} \rightarrow q)
$$

where the occurrence of a certain position state $q$ in 7 is proportional to te probability $\pi(q)$. Equation 8 describes the thermodynamic equilibrium of a molecular system with two (or more) possible states / conformations $q$ and $\widetilde{q}$. The probability of being in state $q$ and switching over to state $\widetilde{q}$ is equal to the probability for the reverse way. For the sufficient and necessary "balance condition" which is less rigorous than Equation 8, see Manousiouthakis \& Deem (1999).

\subsection{Metropolis-Hastings algorithm}

In the following, we use a Metropolis-Hastings type algorithm (Metropolis et al. (1953)) where the transition probability density function $\mathbf{P}$ in Equation 8 is split into two factors

$$
\mathbf{P}(q \rightarrow \widetilde{q})=\mathbf{P}_{p r}(q \rightarrow \widetilde{q}) \mathbf{P}_{\mathrm{ac}}(q \rightarrow \widetilde{q}) .
$$

Here is the corresponding sampling scheme:

- In Equation $9, \mathbf{P}_{\text {pr }}$ is a proposal probability density function, i.e. the probability that after $q \in \Omega$ a position state $\widetilde{q} \in \Omega$ is proposed as candidate for the next step in the Markov chain (see Equation 7).

- With an acceptance probability of $\mathbf{P}_{\mathrm{ac}}$, the next step in the chain is $\widetilde{q}$, with a probability of $1-\mathbf{P}_{\mathrm{ac}}$ the step $q$ is repeated in Equation 7. For a numerical realization of the acceptance probability, one computes a uniformly distributed random number $r \in[0,1]$ and accepts $\widetilde{q}$ if $r \leq \mathbf{P}_{\mathrm{ac}}(q \rightarrow \widetilde{q})$.

With Equation 8 a sufficient condition for a correct sampling according to this scheme is:

$$
\pi(q) \mathbf{P}_{\mathrm{pr}}(q \rightarrow \widetilde{q}) \mathbf{P}_{\mathrm{ac}}(q \rightarrow \widetilde{q})=\pi(\widetilde{q}) \mathbf{P}_{\mathrm{pr}}(\widetilde{q} \rightarrow q) \mathbf{P}_{\mathrm{ac}}(\widetilde{q} \rightarrow q)
$$

For a given (ergodic) proposal probability density function $\mathbf{P}_{\mathrm{pr}}$, a possible choice for $\mathbf{P}_{\mathrm{ac}}$ satisfying the latter equation is for example the Metropolis dynamics:

$$
\mathbf{P}_{\mathrm{ac}}(q \rightarrow \widetilde{q})=\min \left\{1, \frac{\pi(\widetilde{q}) \mathbf{P}_{\mathrm{pr}}(\widetilde{q} \rightarrow q)}{\pi(q) \mathbf{P}_{\mathrm{pr}}(q \rightarrow \widetilde{q})}\right\}
$$

Metropolis dynamics provides a chain of the form of Equation 9 with minimal asymptotic variance and is therefore the most popular one (see Peskun's theorem in Peskun (1973)).

\subsection{Combination of MCMC and MD.}

The transition probabilities $\mathbf{P}(q \rightarrow \widetilde{q})$ doesn't need to have any physical meaning in order to meet Equation 8, but for a good acceptance ratio $\mathbf{P}_{\mathrm{ac}}$, a combination of the Metropolis-Hastings algorithm for Markov chain Monte Carlo integration (MCMC) with molecular dynamics simulations (MD) is useful.

Starting in 1980, a variety of hybrid methods have been developed, which take the advantages of both MD and MCMC (Andersen (1980); Duane et al. (1987)). These so-called HMC algorithms where originally developed for quantum chromo-dynamics, but they have been 
used successfully for condensed-matter systems (Clamp et al. (1994); Forrest \& Suter (1994); Gromov \& de Pablo (1995); Irbäck (1994); Mehlig et al. (1992)) and also for biomolecular simulations (Fischer et al. (1998); Hansmann et al. (1996); Zhang (1999)). HMC combines the large steps of MD in phase space with the property of MCMC to ensure ergodicity by altering orbits in phase space and to eliminate inaccuracies in the numerical computation of the Hamiltonian dynamics. In the following the HMC method is explained according to Fischer (1997).

\subsection{Proposal step}

HMC is a Metropolis algorithm, in which the proposal step $q \rightarrow \widetilde{q}$ is based on a molecular dynamics simulation with simulation length $\tau$. To compute $\widetilde{q}$ out of a given $q$, we first determine a start momentum vector $p \in \mathbb{R}^{3 n}$, where $n$ is the number of atoms. The start momentum vector is taken from the Boltzmann distribution $\eta(p)$ according to the simulation temperature (see Allen \& Tildesley (1987), Section 5.7.2 for algorithmic details). Then, with some numerical integrator a trajectory of total length $\tau$ is computed. The starting point is given by $(q, p)$, let the end point be denoted as $(\widetilde{q}, \widetilde{p})=\Phi_{h}^{\tau}(q, p)$.

Due to determinism in the integration scheme, the probability for a proposition $\mathbf{P}_{\mathrm{pr}}(q \rightarrow \widetilde{q})$ only depends on the choice of the initial momenta $p$, which is $\mathbf{P}_{\operatorname{pr}}(q \rightarrow \widetilde{q}) \propto \exp (-\beta K(p))$.

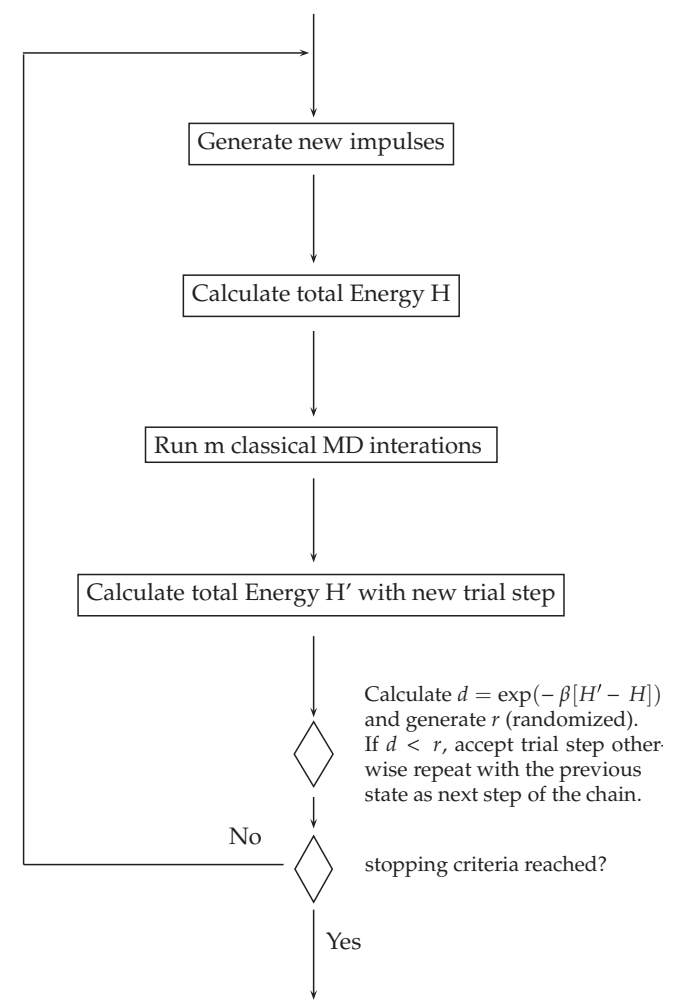

Fig. 2. The HMC Algorithm. 
If the numerical integrator is momentum-reversible, then for the reverse step $\widetilde{q} \rightarrow q$, we have to choose the start momentum vector $-\widetilde{p}$, which transfers the starting point $(\widetilde{q},-\widetilde{p})$ to $(q,-p)$ in time span $\tau$. This means, $\mathbf{P}_{\mathrm{pr}}(\widetilde{q} \rightarrow q) \propto \exp (-\beta K(-\widetilde{p}))$. In Equation 8, the two terms are integrated over the Lebesgue measure $d q \wedge d \widetilde{q}$. After transformation into the momenta space, the left hand side depends on $d q \wedge d p$ and the right had side on $d \widetilde{q} \wedge d \widetilde{p}$. But this does not change anything in Equation 10 because the mapping $(\widetilde{q}, \widetilde{p})=\Phi_{h}^{\tau}(q, p)$ is area preserving. Both measures are equivalent. Inserting these results into 10 yields

$$
\begin{aligned}
\mathbf{P}_{\mathrm{ac}}(q \rightarrow \widetilde{q}) & =\min \left\{1, \frac{\pi(\widetilde{q}) \exp (-\beta K(-\widetilde{p}))}{\pi(q) \exp (-\beta K(p))}\right\} \\
& =\min \left\{1, \frac{\exp (-\beta V(\widetilde{q})) \exp (-\beta K(\widetilde{p}))}{\exp (-\beta V(q)) \exp (-\beta K(p))}\right\} \\
& =\min \{1, \exp (-\beta(H(\widetilde{q}, \widetilde{p})-H(q, p)))\},
\end{aligned}
$$

i.e. the acceptance probability of the HMC proposal step is based on the change of the total energy during a numerical integration of the Hamiltonian. A reversible and area-preserving numerical integrator is necessary and sufficient for a correct sampling. For a rigorous proof, see also Mehlig et al. (1992), Section III.

\subsection{Choice of the numerical integrator}

Table 2 shows some algorithmic details of HMC and related methods. It shows, which Hamiltonian the methods are based upon, the acceptance probabilities and eventually a necessary pointwise re-weighting of the sampling trajectory in order to be mathematically rigorous.

\subsection{1 "Exact" flow}

As we have seen in the derivation of the acceptance probability, a reversible and area-preserving numerical integrator is necessary and sufficient for a correct sampling, no matter how bad its state space solution is. However, if we could apply an "exact" integrator the total energy would be constant during simulation, and therefore, the acceptance probability according to Metropolis dynamics would be 1 . Adaptive integrators can be used with a pre-defined deviation from the exact flow. An example for an adaptive integrator for Hamiltonian dynamics is DIFEX2, an extrapolation method based on Störmer discretization, see Deuflhard (1983) and Deuflhard (1985). For DIFEX2, area-preservation and reversibility cannot be shown directly, but as the extrapolation method approximates the real flow, it inherits these properties from $\Phi^{\tau}$. Other adaptive integration methods and Fortran codes can be found in the book of Hairer et al. (1993). See also the first row of Table 2.

\begin{tabular}{|l|l|l|l|}
\hline & Hamiltonian & accept & re-weight \\
\hline \hline "exact" flow & $H$ & 1 & no \\
\hline orig. HMC & $H$ & $e^{-\beta \Delta H}<1$ & no \\
\hline SHMC (idea) & $\widetilde{H}$ & 1 & $\widetilde{H} \rightarrow H$ \\
\hline SHMC & $\widetilde{H}_{a p p}$ & $e^{-\beta \Delta \widetilde{H}_{a p p}} \approx 1$ & $\widetilde{H}_{a p p} \rightarrow H$ \\
\hline
\end{tabular}

Table 2. Possible approaches for a correct sampling according to the HMC method. Algorithmic consequences. 


\subsubsection{Original HMC}

Instead of solving the real dynamics, one can apply an arbitrary area-preserving and reversible integrator. In this case, the mean acceptance ratio decreases exponentially with system size $n$ and the time step discretization $h$ in the numerical integration $\Phi_{h}^{\tau}$. See Gupta et al. (1990) and Kennedy \& Pendleton (1991) for an analytic study of the computational cost of HMC. It is a general opinion that HMC methods are only suitable for small molecular systems (see e.g. Section 14.2 in Frenkel \& Smit (2002)). The reason is that in order to keep the mean acceptance probability constant for increasing system size, the time step $h$ of the symplectic integrator has to decrease accordingly. Instead of time step refinements, one can also increase the order of the integration method. Using Hamilton's principle of a stationary action integral, Wendlandt \& Marsden (1997) derived a systematical scheme for creating so-called variational integrators. With these higher-order numerical integrators the acceptance ratio of HMC can be improved due to better approximation properties. By omitting the constant step size $h$ in variational integrators, one can even get symplectic and energy preserving integration schemes for the price of lower numerical efficiency. For an excellent overview of these methods, see Lew et al. (2004). See also the second row of Table 2.

\subsubsection{Shadow HMC (SHMC)}

Another approach uses the fact that some symplectic numerical integrator solves the dynamics of a modified Hamiltonian $\widetilde{H}$ exactly (see Hairer et al. (2004) or Skeel \& Hardy (2002)). If one accepts each proposal step of the numerical dynamics simulation, this is like computing the density of the modified Hamiltonian (see third row of Table 2). In order to get the right distribution in configuration space, one has to re-weight the resulting position states accordingly. This is only possible, if the modified Hamiltonian is known. Fortunately, $\widetilde{H}$ can be approximated up to arbitrary accuracy. For algorithmic details, see Izaguirre \& Hampton (2004).

\subsubsection{Approximated SHMC}

Approximating the modified Hamiltonian as exactly as possible, is numerically expensive. Therefore, one would like to truncate the Taylor expansion of $\widetilde{H}$ after a finite number of terms in order to yield $\widetilde{H}_{\text {app }}$. This method is part of the TSHMC method of Akhmatskaya \& Reich (2004). Again, an acceptance rule is introduced which zeroes out the numerical error of truncation (see the last row of Table 2). This method seems to be very promising for larger molecules, because the acceptance probability is almost 1 , the method is mathematically rigorous and numerically efficient (extra cost for computation of $\widetilde{H}_{\text {app }}$ is negligible).

\section{Example: Brominated flame retardant hexabromocyclododecane}

$1,2,5,6,9,10$-Hexabromocyclododecane (HBCD) is a widely used additive brominated flame retardant (BFR) to plastic materials as upholstery textiles, styrene-acrylonitrile resins or polystyrene foams (EPS, XPS) for the building sector with fractional percentages varying between 0.8 and 4\% (Barda et al. (1985); de Witt (2002); Janak et al. (2005)). In the face of a world market demand of about 22000 metric tons in the year 2003 (Köppen et al. (2008)), HBCD is among a the most popular BFRs, especially in Europe (Janak et al. (2005)).

However, it is regarded as a persistent organic pollutant (POP) and has been detected increasingly in the environment during the last decades, e. g. in sewage sludges and sediments (see de Wit et al. (2006); Hale et al. (2006); Vos et al. (2003)) as well as in diverse tissues of both 
terrestric and aquatic organisms (Janak et al. (2005); Tomy et al. (2004)). Even in the lipid phase of human breast milk of women from diverse european countries and the USA traces of HBCD and other BFR have been detected (Covaci et al. (2006); Johnson-Restrepo et al. (2008)).

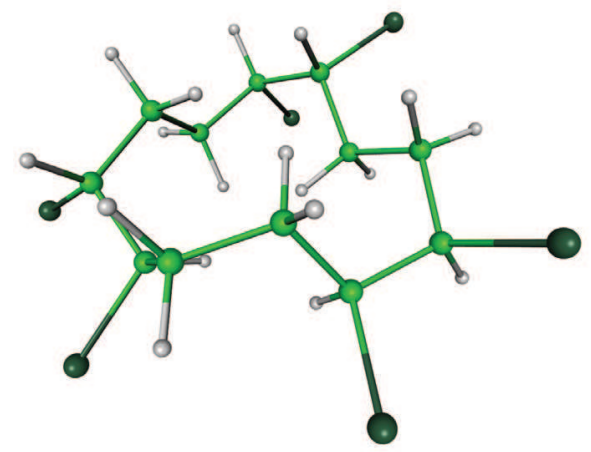

Fig. 3. 3-dimensional chemical structure of $(-)-\alpha-H B C D$ with six dark colored bromine atoms.

\subsection{Technical and structural properties of HBCD}

Technical HBCD is produced by bromination of the precursor 1,5,9-cyclododecatriene (CDT) unavoidably resulting in the formation of six stereocenters (Becher (2005); Heeb et al. (2004)). Theoretically, this yields 16 different HBCD diastereomers, six pairs of enantiomers and four mesoforms (Heeb et al. (2008)). But starting especially with the precursor (1Z,5E,9E)-CDT, the technical production results in a racemic mixture of three enantiomeric pairs $( \pm)-\alpha-,-\beta-$, $-\gamma$-HBCD) and two mesoforms $(\delta-, \epsilon$-HBCD). The latter two will be neglected in the present work due to their negligible amounts in both the technical and the environmental mixture. The technical composition containing ( + -) $\gamma$-HBCD mainly (Janak et al. (2005)) is presented in Table 3. See Fig. 5 for a graphical overview of the six main HBCD isomers. They are all characterized by a $(R, R)$ or $(S, S)$ configuration on their $\mathrm{C}_{1} \mathrm{Br}-\mathrm{C}_{2} \mathrm{Br}$-moiety and an $(S, R)$ or $(R, S)$ configuration on the $\mathrm{C}_{5} \mathrm{Br}-\mathrm{C}_{6} \mathrm{Br}-$ and $\mathrm{C}_{9} \mathrm{Br}-\mathrm{C}_{10} \mathrm{Br}$-moieties. On the former moiety, $\alpha-$ and $\gamma$-HBCD feature $C_{2}$-symmetry, implying identical energetical properties for the latter two moieties, whereas the $\beta$-diastereomer does not possess any rotational symmetries.

In contrast to the technical mix, a completely different composition was found in the abiotic and biotic environment, which are always by far predominated by $\alpha$-HBCD (Becher (2005); Heeb et al. (2008); Tomy et al. (2004)) with slightly differing concentrations and even enantioselective accumulation (Tomy et al. (2004)). Certainly, these observations raise the question of their cause. On the one hand, this might arise from variability in physicochemical properties such as dipole moment or solubility in water. The latter with respect to $\alpha-, \beta-$

\begin{tabular}{|c|c|c|c|}
\hline Diastereomer & CIP nomenclature of both enantiomers & $\begin{array}{c}\text { Molar fraction } \\
\text { Technical }\end{array}$ & Equilibrium \\
\hline \hline$( \pm)-\alpha-\mathrm{HBCD}$ & $1 \mathrm{~S}, 2 \mathrm{~S}, 5 \mathrm{R}, 6 \mathrm{~S}, 9 \mathrm{~S}, 10 \mathrm{R}-\mathrm{HBCD}, 1 \mathrm{R}, 2 \mathrm{R}, 5 \mathrm{~S}, 6 \mathrm{R}, 9 \mathrm{R}, 10 \mathrm{~S}-\mathrm{HBCD}$ & $10-13 \%$ & $78 \%$ \\
\hline$( \pm)-\beta$-HBCD & $1 \mathrm{~S}, 2 \mathrm{~S}, 5 \mathrm{R}, 6 \mathrm{~S}, 9 \mathrm{R}, 10 \mathrm{~S}-\mathrm{HBCD}, 1 \mathrm{R}, 2 \mathrm{R}, 5 \mathrm{~S}, 6 \mathrm{R}, 9 \mathrm{~S}, 10 \mathrm{R}-\mathrm{HBCD}$ & $1-12 \%$ & $14 \%$ \\
\hline$( \pm)-\gamma-\mathrm{HBCD}$ & $1 \mathrm{R}, 2 \mathrm{R}, 5 \mathrm{R}, 6 \mathrm{~S}, 9 \mathrm{~S}, 10 \mathrm{R}-\mathrm{HBCD}, 1 \mathrm{~S}, 2 \mathrm{~S}, 5 \mathrm{~S}, 6 \mathrm{R}, 9 \mathrm{R}, 10 \mathrm{~S}-\mathrm{HBCD}$ & $75-89 \%$ & $8 \%$ \\
\hline
\end{tabular}

Table 3. CIP names of HBCD isomers and their fraction in the technical mix and at equilibrium. 


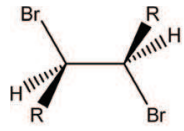

$(1 \mathrm{R}, 2 \mathrm{R})$

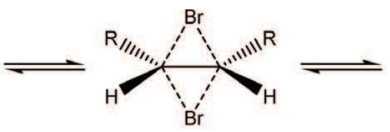

Br

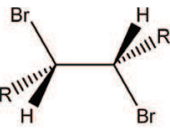

$(1 \mathrm{~S}, 2 \mathrm{~S})$

Fig. 4. Cyclic-concerted interconversion mechanism. A necessary condition for the quantum-chemically induced process is the anti positioning of two vicinal bromine atoms which leads to double-bonded bromines and five-bonded carbons during transition (center structure) caused by a nucleophilic attack of bromine. The interconversion results in a change of the involved chiralities.

and $\gamma$-HBCD is relatively low and was measured to be $48.8,14.7$, and $2.1 \mu \mathrm{g} / \mathrm{L}$, respectively (Hunziker et al. (2004)).

On the other hand, differing diastereomeric ratios might be triggered by stereoselective uptake and metabolism. Indeed, there seems to be strong evidence for a biologically induced interconversion of HBCD stereoisomers (Hamers et al. (2006); Law et al. (2006a)). According to Vos et al. (2003), Hamers et al. (2006), and Meerts et al. (2000), some BFRs such as HBCD or its metabolite pentabromocyclododecaene (PBCD) are suspected to cause endocrine disruption

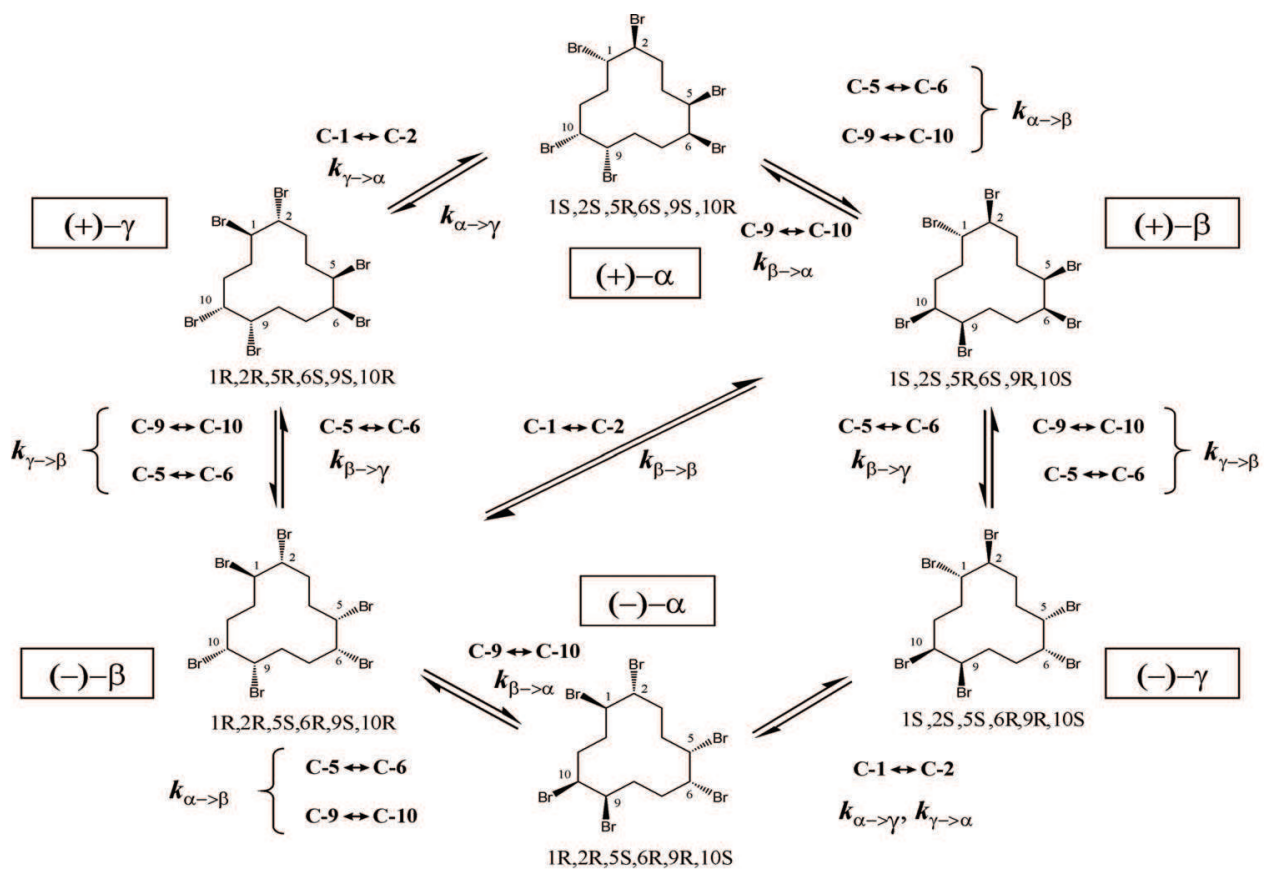

Fig. 5. Graphical overview of all possible interconversions between any connected two of the six main HBCD diastereomers taken from Köppen et al. (2008). The bromine anti conformation of any $\mathrm{C}_{i} \mathrm{Br}-\mathrm{C}_{i+1} \mathrm{Br}$-moiety of the starting diastereomers triggers the inversion to another diastereomer depending on the couple of concerned bromine atoms, i.e. depending on the value of index $i$ with $i \in\{1,5,9\}$. This transition process with rate $k_{x \rightarrow y}$ is denoted as cyclic-concerted interconversion. 
due to competition with thyroxine $\left(\mathrm{T}_{4}\right)$ for binding to the human transthyretin receptor (hTTR). Recently, Schriks et al. (2006) observed effects on the cell proliferation of Xenopus laevis induced by HBCD and another BFR denoted as BDE206 (brominated diphenyl ether).

Temperatures above $160^{\circ} \mathrm{C}(433 \mathrm{~K})$, which is close to the melting point of crystalline HBCD at $188-191^{\circ} \mathrm{C}$, induce a isomerization process denoted as cyclic-concerted interconversion (Köppen et al. (2008)) described in detail below. As shown in Table 3, the equilibrium distribution of the HBCD diastereomers after thermal rearrangement is dominated by $\alpha$-HBCD with a fraction of $78 \%$ followed by $\beta$ - and $\gamma$-HBCD with percentages of $13 \%$ and $9 \%$, respectively (Peled et al. (1995)). Temperatures exceeding $200^{\circ} \mathrm{C}$ lead to HBCD decomposition (Barontini et al. (2003)).

\subsection{Interconversion kinetics of HBCD}

Any pair of vicinal bromine atoms each bonded with a chiral carbon $\left(\mathrm{C}_{i} \mathrm{Br}-\mathrm{C}_{i+1} \mathrm{Br}\right.$-moiety) is able to undergo a stereo-isomerization process denoted as cyclic-concerted interconversion resulting in complementary chirality (Fig. 4) of both involved carbons (Köppen et al. (2008)). The transition state which is quantum-chemically motivated requires both bromine atoms to be anti-positioned and to form a second bond whith the respective vicinal chiral carbon atoms which switch from $\mathrm{sp}_{3}$ to $\mathrm{sp}_{2}$ hybridization and to a five-bonded state during transition (Fig. 4 , center graphic).

Fig. 5 shows all possible interconverions between the six main diastereomers depending on the concerned $\mathrm{C}_{i} \mathrm{Br}-\mathrm{C}_{i+1} \mathrm{Br}$-moiety with $i \in\{1,5,9\}$. For example, if the $\mathrm{C}_{1} \mathrm{Br}-\mathrm{C}_{2} \mathrm{Br}$-moiety of $(+)-\gamma$-HBCD forms a anti-conformation of the bromines, which will happen with a certain probability in terms of statistical thermodynamics, the chirality of $\mathrm{C}_{1} \mathrm{Br}-\mathrm{C}_{2} \mathrm{Br}$ will change from $(1 \mathrm{R}, 2 \mathrm{R})$ to $(1 \mathrm{~S}, 2 \mathrm{~S})$ resulting in $(+)-\alpha$-HBCD.

As already mentioned above, the system of diastereomers moves towards a thermodynamical equilibrium dominated by $(+)-\alpha$-HBCD. Recently, rate constants $k_{x \rightarrow y}$ have been determined by Köppen et al. (2008) at $160^{\circ} \mathrm{C}$ via high performance liquid chromatography (HPLC) using a chiral column. The results are presented in Table 4 and graphically in Fig. 6. It is worth mentioning that the rates are supposed to be the same for the enantiomeric counterparts in this thermally induced process. The fastest interconversion after these results is the one from $\gamma$-HBCD to $\alpha$-HBCD, closely followed by the interconversion from $\beta$-HBCD to its enantiomeric counterpart. Both reactions occur on the $C_{1}-C_{2}$-moiety. By far, the lowest rate was measured in case of the interconversion from $\alpha$ - to $\beta$-HBCD.
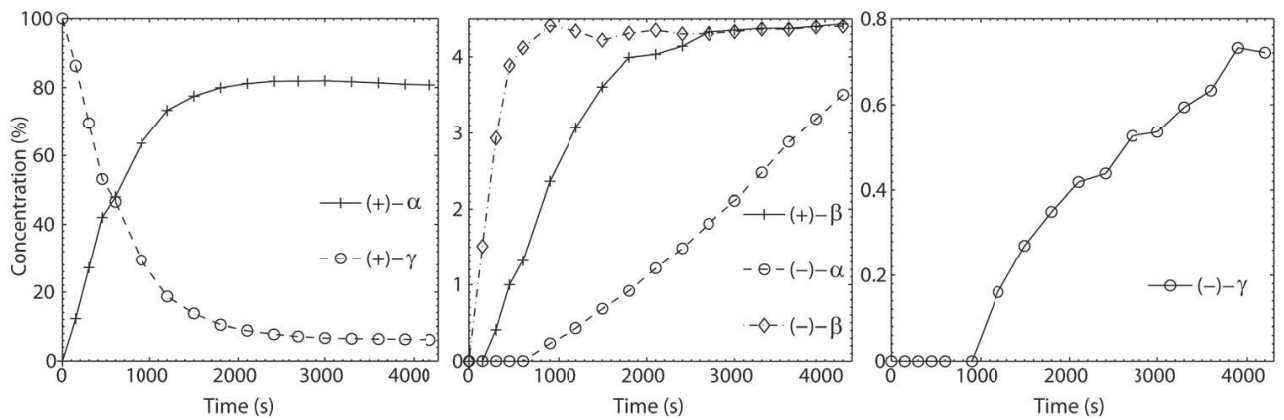

Fig. 6. Experimental determination on the isomerization of $\mathrm{HBCD}$ at $160^{\circ} \mathrm{C}$ starting with $100 \%(+)-\gamma$-HBCD. The tests were carried out by Köppen et al. (2008). 


\begin{tabular}{|c|c|c|}
\hline Interconversion & $\mathrm{C}_{i} \mathrm{Br}-\mathrm{C}_{i+1} \mathrm{Br}-\mathrm{moiety}$ & Rate constant $k\left[\frac{\mathrm{mol}(\%)}{\mathrm{s}}\right]$ \\
\hline \hline$k_{\alpha \rightarrow \beta}$ & $\mathrm{C}_{5}-\mathrm{C}_{6} / \mathrm{C}_{9}-\mathrm{C}_{10}$ & $1.88 \pm 0.01 \times 10^{-5}$ \\
\hline$k_{\alpha \rightarrow \gamma}$ & $\mathrm{C}_{1}-\mathrm{C}_{2}$ & $1.42 \pm 0.01 \times 10^{-4}$ \\
\hline$k_{\beta \rightarrow \alpha}$ & $\mathrm{C}_{9}-\mathrm{C}_{10}$ & $1.20 \pm 0.01 \times 10^{-4}$ \\
\hline$k_{\beta \rightarrow \beta}$ & $\mathrm{C}_{1}-\mathrm{C}_{2}$ & $1.10 \pm 0.03 \times 10^{-3}$ \\
\hline$k_{\beta \rightarrow \gamma}$ & $\mathrm{C}_{5}-\mathrm{C}_{6}$ & $1.70 \pm 0.01 \times 10^{-4}$ \\
\hline$k_{\gamma \rightarrow \alpha}$ & $\mathrm{C}_{1}-\mathrm{C}_{2}$ & $1.50 \pm 0.01 \times 10^{-3}$ \\
\hline$k_{\gamma \rightarrow \beta}$ & $\mathrm{C}_{5}-\mathrm{C}_{6} / \mathrm{C}_{9}-\mathrm{C}_{10}$ & $1.46 \pm 0.01 \times 10^{-4}$ \\
\hline
\end{tabular}

Table 4 . Rate constants $k_{y \rightarrow z}$ with $y, z \in\{\alpha, \beta, \gamma\}$ for the cyclic-concerted interconversion process of $\mathrm{HBCD}$ at $160^{\circ} \mathrm{C}$.

\section{Simulation of HBCD interconversion rates}

As already mentioned in section 4, the six main HBCD diastereomers underly a isomerization process at temperatures between 433 and $473 \mathrm{~K}$ denoted as cyclic-concerted interconversion (see Fig. 5) which is quantum-chemically motivated and results in complementary chirality of both concerned vicinal brominated carbon atoms. Nevertheless, this section presents an approach for estimating transition rates of such processes in terms of classical mechanics. It is due to the matter of time scale and the complexity of electronic densities, that the interconversion can hardly be investigated by quantum-chemical methods without enormous computational costs.

Due to high rotational barriers, high-temperature simulations are inevitable in order to avoid trapping effects. Hence, the adoption of these results to lower temperatures requires a reweighting strategy which has been developed within the framework of these investigations. Afterwards, the interconversion rates are approximated on the basis of free energy calculations in combination with the Arrhenius equation and by applying the transition state theory (TST). Fortunately, experimental data of the interconversion kinetics is available and will be compared to the theoretical results obtained here.

\subsection{Classical model for the interconversion}

A necessary condition for the transition state of interconversion processes is the anti-positioning of two vicinal bromine atoms in liquid phase, that we will call an "active state". It is assumed to occur more likely with lower rotational barriers between anti and gauche conformations and vice versa. The rate of the transition is considered to depend on both, the probability of such an activated state, which can be described in terms of classical thermodynamics and the velocity of the quantum-chemically motivated transition itself. The latter may simply be neglected due to the assumption of its identity in case of all HBCD diastereomers. Therefore, a qualitative definition of interconversion rates in terms of free energy differences only between two subsets $\Omega_{\text {anti }}$ and $\Omega_{\text {gauche }} \in \mathbb{R}^{3 n}$ of the conformational space $\Omega \in \mathbb{R}^{3 n}$ turns out to be a suitable approach.

Initially, a sampling of the conformational space had to be performed with the HMC method at an artificially high temperature $T=1500 \mathrm{~K}$ (i. e. with the Boltzmann factor $\beta_{0}=0.0802 \mathrm{~mol} / \mathrm{kJ}$ and in vacuum, neglecting mutual interactions with other HBCD molecules or with a solvent such as water and therefore, reducing computational costs. We decided to utilize the Merck molecular force celd (mmff) designed by Halgren (1996) for our simulations. For each diastereomer, five trajectories were constructed each consisting of $100.000 \mathrm{MC}$ steps with $60 \mathrm{MD}$ steps per MC step. The MD integration step was set to $1.3 \mathrm{fs}$. Convergence was 
checked in accordance with Gelman \& Rubin (1992) on the basis of the five Markov chains. The conjugate gradient (CG) minimization method (Hestenes \& Stiefel (1952)) was used for energy optimization of the sampled geometries in order to identify all local and thus, global minima. The free energy $A(\beta)$ is defined in terms of the partition function

$$
A(\beta)=-\frac{1}{\beta} \ln \left(\int_{\Omega} \exp (-\beta V(q)) \mathrm{d} q\right) .
$$

Note, that the kinetic part $K(p)$ of the total energy $H(q, p)$ is missing due to the assumption of its idendity for both conformational subsets at identical temperature. In this section, we will only consider the potential energy fraction $V(q)$ of the separable Hamiltonian. It is not possible to approximate the integral in Equation 12; however, free energy differences $\Delta_{\mathrm{ga}} A(\beta)$ (a = anti,g $=$ gauche $)$ of two conformational subsets $\Omega_{\mathrm{anti}}$ and $\Omega_{\text {gauche }}$ may be approximated. Due to importance sampling (compare Equation 5 with 6), the number of geometries containing anti- $\left(N_{\text {anti }}\right)$ and gauche-conformation ( $\left.N_{\text {gauche }}\right)$, respectively, according to the $\mathrm{C}_{i} \mathrm{Br}-\mathrm{C}_{i+1} \mathrm{Br}$-moiety under consideration is sufficiant in order to approximate

$$
\Delta_{\mathrm{ga}} A(\beta) \approx-\frac{1}{\beta} \ln \left(\frac{N_{\text {gauche }}}{N_{\text {anti }}}\right) .
$$

A dihedral angle $\theta$ between two vicinal bromine atoms was defined as anti if $|\theta|>120^{\circ}$, i. e. if the lower bromine atom remained in the grey-coloured segment as depicted in Fig. 7, whereas an angle $120 \geq \theta \geq-120^{\circ}$ was defined as gauche.

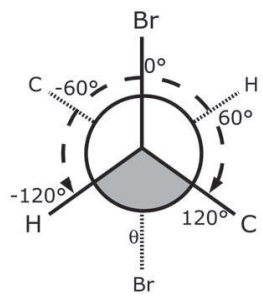

Fig. 7. For the interconversion anlysis, the bromine's conformation was defined as anti if the dihedral $\theta$ was greater than $120^{\circ}$ and less than $-120^{\circ}$ (lower bromine within the grey segment).

These free energy differences were calculated for each $\mathrm{C}_{i} \mathrm{Br}-\mathrm{C}_{i+1} \mathrm{Br}$-moiety of all (+)-HBCD enantiomers. The lower the free energy difference, the more the state indicated by the first index $(g=$ gauche $)$ is preferred.

In the followings, we will additionally need another thermodynamic quantity derived from the simulated data, namely the mean potential energy $\langle V\rangle$ defined as a function of temperature $\beta$

$$
\langle V\rangle(\beta)=\int_{\Omega} V(q) \frac{\exp (-\beta V(q))}{\int_{\Omega} \exp (-\beta V(q)) \mathrm{d} q} \mathrm{~d} q
$$

which can be approximated by the arithmetic mean of all potential energy values from the HMC sampling. Again, the energy difference $\Delta_{\text {ga }}\langle V\rangle$ was determined for each $\mathrm{C}_{i} \mathrm{Br}-\mathrm{C}_{i+1} \mathrm{Br}$-moiety of all $(+)$-HBCD enantiomers, after having partitioned the conformational space $\Omega$ into gauche and anti subsets and having calculated their respective mean potential energies. 


\subsection{Thermodynamical energy reweighting}

The HMC sampling had been performed at temperature $T=1500 \mathrm{~K}\left(\beta_{0}=0.0802 \mathrm{~mol} / \mathrm{kJ}\right)$ due to convergence reasons. However, we are interested in the energy distribution at $T=$ $433 \mathrm{~K}\left(\beta_{1}=0.2778 \mathrm{~mol} / \mathrm{kJ}\right)$ which marks the melting point of HBCD. Usually, reweighting is understood in a point-wise way, reweighting the complete distribution to the temperature of interest and, therefore, statistically weighting up a relatively small number of points in the overlap region of both the high- and the low-temperature distribution (see grey coloured area in Fig. 8).

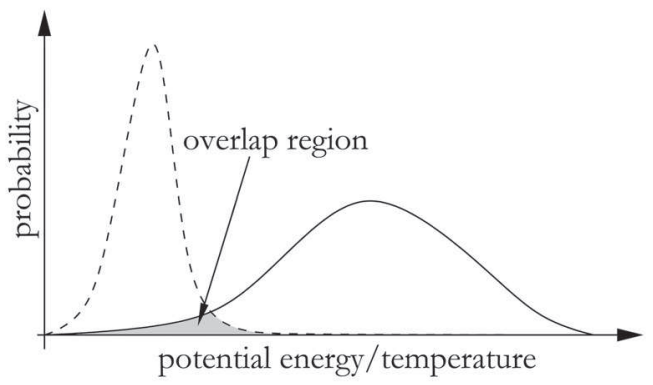

Fig. 8. Overlap region between high and low potential energy distributions relevant for point-wise reweighting.

Here, we apply a thermodynamical approach instead of a point-wise one, making use of the temperature-dependency of the distribution's mean value, i.e. its dependency on $\beta$. In analogy to the mean kinetic energy $\langle K\rangle$, we assume a linear dependency of the mean potential energy $\langle V\rangle$ on the temperature $T$ (i.e. on $\beta^{-1}$ )

$$
\Delta_{\mathrm{ga}}\langle V\rangle(\beta)=\frac{\left[\Delta_{\mathrm{ga}}\langle V\rangle\left(\beta_{0}\right)-\Delta_{\mathrm{ga}}\langle V\rangle(\infty)\right] \beta_{0}}{\beta}+\Delta_{\mathrm{ga}}\langle V\rangle(\infty)
$$

$\Delta_{\mathrm{ga}} V(\infty)$ denotes the difference of the global minimal energies of both subspaces at absolute zero $T=0 \mathrm{~K} \Leftrightarrow \beta=\infty$. Since the system moves towards the global optimum with decreasing temperature, a conjugate gradient minimization was applied to the complete canonical high-temperature ensembles of $\mathrm{HBCD}$ geometries. Again, each $\mathrm{C}_{i} \mathrm{Br}-\mathrm{C}_{i+1} \mathrm{Br}$-moiety of all $(+)$-HBCD enantiomers underwent this procedure after having been separated according to their anti/gauche-isomerization. In addition and via Equation 15, mean potential energies and their differences for each $\mathrm{C}_{i} \mathrm{Br}-\mathrm{C}_{i+1} \mathrm{Br}$-moiety can now be interpolated to the temperature of interest which is the melting point of $\mathrm{HBCD}$ at $433 \mathrm{~K}$. See Fig. 9 for a graphical representation of the linear model.

However, in order to determine interconversion rates as described in the next section, free energy differences for each $\mathrm{C}_{i} \mathrm{Br}-\mathrm{C}_{i+1} \mathrm{Br}$-moiety at the desired temperature needed to be estimated as well. This was achieved by first differentiating Equation 12 and inserting Equation 14

$$
\frac{\mathrm{d}}{\mathrm{d} \beta} \Delta_{\mathrm{ga}} A(\beta)=-\frac{1}{\beta} \Delta_{\mathrm{ga}} A(\beta)+\frac{1}{\beta} \Delta_{\mathrm{ga}}\langle V\rangle(\beta) .
$$

Inserting the linear model (Equation 15) instead of the last term of Equation 16 leads to the ordinary differential equation

$$
\frac{\mathrm{d}}{\mathrm{d} \beta} \Delta_{\mathrm{ga}} A(\beta)=-\frac{1}{\beta} \Delta_{\mathrm{ga}} A(\beta)+\frac{\left[\Delta_{\mathrm{ga}}\langle V\rangle\left(\beta_{0}\right)-\Delta_{\mathrm{ga}} V(\infty)\right] \beta_{0}}{\beta^{2}}+\frac{\Delta_{\mathrm{ga}} V(\infty)}{\beta} .
$$




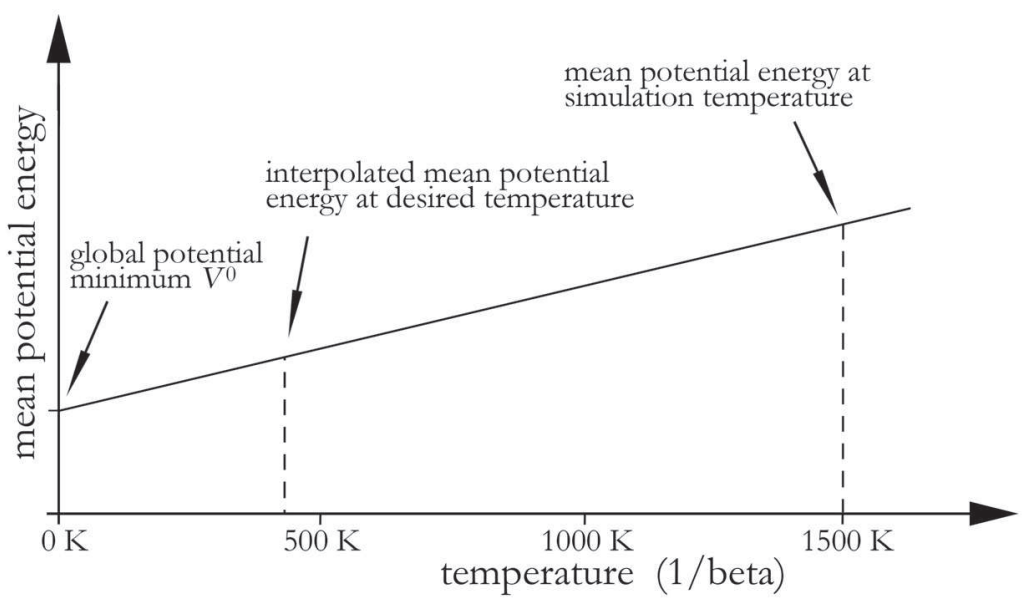

Fig. 9. A linear model for the thermodynamical reweighting of mean potential energies to temperatures between the one used for high-temperature simulations and absolute zero.

which can be solved analytically, such that

$$
\begin{aligned}
\Delta_{\mathrm{ga}} A(\beta) & =\frac{\beta-\beta_{0}}{\beta} \Delta_{\mathrm{ga}}\langle V\rangle(\infty) \\
& +\frac{\beta_{0}}{\beta} \ln \left(\frac{\beta}{\beta_{0}}\right)\left[\Delta_{\mathrm{ga}}\langle V\rangle\left(\beta_{0}\right)-\Delta_{\mathrm{ga}}\langle V\rangle(\infty)\right]+\frac{\beta_{0}}{\beta} \Delta_{\mathrm{ga}} A\left(\beta_{0}\right) .
\end{aligned}
$$

Equation 18 allows to interpolate free energy differences for temperatures such as the desired one at $T=433 \mathrm{~K}$.

\subsection{Rate matrix and interconversion kinetics}

Basically, the estimation of interconversion rates rested upon the combination of the Arrhenius equation (Göpel \& Wiemdörfer (2000)) with the transition state theory (TST) (Weber (2007)). According to Arrhenius, the reaction rate $k$ depends on the temperature $T$ (respectively $\beta$ ) and on the activation energy $E_{A}$ as follows

$$
k=A \cdot \exp \left(-\beta E_{A}\right)
$$

with a prefactor $A$. In this work, the activation energy for the interconversion process at a certain $\mathrm{C}_{i} \mathrm{Br}-\mathrm{C}_{i+1} \mathrm{Br}$-moiety requiring anti conformation was approximated by the free energy difference between the respective subspaces of the conformational space, due to an obvious dependency of the interconversion rate $k$ from the free energy difference $\Delta_{a g} A=-\Delta_{\text {ga }} A$. In other words, the higher the free energy difference between the gauche and anti subspace is, the more likely the system is to form anti conformations and, therefore, the larger the interconversion rate will be. Hence, we propose this correlation such that

$$
k \propto \exp \left(-\beta \Delta_{\mathrm{ag}} A\right)
$$

neglecting the prefactor from Equation 19 which needs to be determined experimentally and marks an upper bound for the maximal reaction rate. As we do not expect to gain quantitative, but qualitative interconversion rates, the prefactor does not play any role for our purposes. 
These rates were used now to fill up non-zero entries of the squared matrix $K \in \mathbb{R}^{6 \times 6}$ with one dimension per HBCD diastereomer in the order $(+)-\alpha,(+)-\beta,(+)-\gamma,(-)-\alpha,(-)-\beta$, $(-)-\gamma$. For example, the element of the third row $((+)-\gamma=$ educt $)$ and the first column $((+)-\alpha=$ product $)$ of $K$ is determined by

$$
k_{3,1}=k_{(+) \gamma \rightarrow(+) \alpha}=\exp \left(-\beta \Delta_{\mathrm{ag}} A\right)
$$

where $\Delta_{\mathrm{ag}} A$ is the free energy difference for anti and gauche conformations of the according $\mathrm{C}_{1} \mathrm{Br}-\mathrm{C}_{2} \mathrm{Br}$-moiety, derivable from Fig. 5. Note, that the interconversion from $\alpha$ - or $\gamma$ - to $\beta$-HBCD is associated with two $\mathrm{C}_{i} \mathrm{Br}-\mathrm{C}_{i+1} \mathrm{Br}$-moieties $\left(\mathrm{C}_{5} \mathrm{Br}-\mathrm{C}_{6} \mathrm{Br}\right.$ and $\left.\mathrm{C}_{9} \mathrm{Br}-\mathrm{C}_{10} \mathrm{Br}\right)$. Here, both respective free energy differences must be summed up before being inserted into Equation 21.

$K$ is a stochastic matrix with the row sums needing to be scaled to 1 . It is also denoted as embedded Markov chain playing a central role in the TST, particularly for the computation of the rate matrix $Q \in \mathbb{R}^{6 \times 6}$ we are interested in

$$
Q=R(K-i d)
$$

where id denotes the six-dimensional unit matrix and $R \in \mathbb{R}^{6 \times 6}$ the diagonal matrix of rate facors (Kijima (1997); Weber (2007)). $Q$ is well-known from the first order rate equation

$$
\frac{\mathrm{d} x(t)}{\mathrm{d} t}=Q^{\top} x(t)
$$

describing changes of the concentration vector $x \in \mathbb{R}^{6}$ over time $t$ using the (transposed) rate matrix $Q$. In case of equilibrium concentrations $x(t)=\pi(t)$ at the systems steady-state, Equation 23 becomes

$$
\frac{\mathrm{d} \pi(t)}{\mathrm{d} t}=Q^{\top} \pi(t)=0 \Longleftrightarrow \pi^{\top} Q=0
$$

This information is necessary for the computation of the matrix $R$ of rate factors which is the last unknown quantity in Equation 22. For this purpose, the right-hand side of equation 22 was inserted into $Q$ of the second part of Equation 24 resulting in

$$
\pi^{\top} R(K-i d)=0
$$

Little manipulations of Equation 25 lead us to the eigenproblem

$$
r^{\top} \Pi(K-i d)=0 \cdot r^{\top}
$$

where the diagonal elements of the diagonal matrix $R$ have been transferred into an ordinary vector $r$ and vice versa in case of the steady-state distribution vector $\pi$ which was transformed into a diagonal matrix $\Pi$. This is a sophisticated way to solve rate factors $r$ (and hence, $R$ and $Q)$, handling them as an eigenvector of the eigenvalue zero. A simulation of these theoretical interconversion kinetics can be performed by solving the differential Equation 23 such that

$$
x(\tau)=x(0) \exp (\tau Q)
$$

starting at an initial distribution $x(0)$ and iterating over a time $\operatorname{span} t=\tau$. All calculations have been performed with the software Matlab. 


\section{Results and discussion}

\subsection{HMC-sampling of HBCD diastereomers}

Global minima of the six major HBCD diastereomers' potential energies are listed in Table 5. Expectedly, the optimal energies are identical for each pair of enantiomers, affirming a sufficient convergence as indicated by the Gelman and Rubin criterion. In contrast to 100.000 HMC steps, a first run with 10.000 steps had turned out to be insufficient to achieve convergence in spite of the high simulation temperature of $1500^{\circ} \mathrm{C}$.

Already the simple results from energy minimized samplings considerably support observations of a predominating $\alpha$-HBCD diastereomer in the environment Becher (2005); Janak et al. (2005); Tomy et al. (2004), in spite of the technical mix mainly being composed of $(+)-\gamma$-HBCD Peled et al. (1995). A comparison of experimentally determined diastereomeric fractions after a thermal rearrangement $(78 \% \alpha, 14 \% \beta, 8 \% \gamma)$ from Table 3 with global potential energy minima $(\alpha: 238.7 \mathrm{~kJ} / \mathrm{mol}, \beta: 249.5 \mathrm{~kJ} / \mathrm{mol}, \gamma: 256.7 \mathrm{~kJ} / \mathrm{mol})$ from Table 6 shows a clear correlation and justifies the predominance of $\alpha$-HBCD at the thermodynamic equilibrium.

\begin{tabular}{|c|c|c||c|c|c|}
\hline Diastereomer & Global minimum & $\frac{\mathrm{kJ}}{\mathrm{mol}}$ & Diastereomer & Global minimum & $\frac{\mathrm{kJ}}{\mathrm{mol}}$ \\
\hline \hline$(+)-\alpha-\mathrm{HBCD}$ & 238.7 & $(-)-\alpha$-HBCD & 238.7 \\
\hline$(+)-\beta-\mathrm{HBCD}$ & 249.5 & $(-)-\beta-\mathrm{HBCD}$ & 249.5 \\
\hline$(+)-\gamma-\mathrm{HBCD}$ & 256.7 & $(-)-\gamma-\mathrm{HBCD}$ & 256.7 \\
\hline
\end{tabular}

Table 5. Global minima of the six main HBCD diateromers' potential energies at $1500 \mathrm{~K}$.

In addition, global potential energy minima have been determined for both conformational subsets (anti and gauche) of each $\mathrm{CBr}-\mathrm{CBr}$-moiety (Table 6). As expected, symmetries were found in the global minima of enantiomeric counterparts of HBCD (same optimum values for $(+)$ - and (-)-enantiomer) as well as in identical optima for potential energy differences $\left(V^{0}\right)$ associated with $\mathrm{C}_{5} \mathrm{Br}-\mathrm{C}_{6} \mathrm{Br}-$ and $\mathrm{C}_{9} \mathrm{Br}-\mathrm{C}_{10} \mathrm{Br}$-moieties of $\alpha$ - and $\gamma$-HBCD as a result of their configurational $\mathrm{C}_{2}$-symmetry. Additionally, all energy differences concerning these two moieties are pretty different from those of the $\mathrm{C}_{1} \mathrm{Br}-\mathrm{C}_{2} \mathrm{Br}$-moiety which is by reason the fact that an anti conformation of bromine atoms is energetically much more favorable on an $(R, R)$ or $(S, S)$ configurated moiety such as the latter mentioned one than on an $(S, R)$ or $(R, S)$ configuration given in case of the two other moieties.

The results in Table 6 allow a qualitative estimation of the disposition to undergo the interconversion mechanism. The higher the Boltzmann prpbability ratio between the minimum of the anti conformation and the corresponding gauche conformation's minimum is, the more likely this molecule will interconvert at the respective $\mathrm{CBr}-\mathrm{CBr}$-moiety. After these results, for example the interconversion from $(+)-\gamma-\mathrm{HBCD}$ to $(+)-\alpha-\mathrm{HBCD}$ (and analogously for their enantiomeric counterparts) with a Boltzmann ratio of 1 is by far the fastest reaction or rather the most probable one. In contrast, the probability of the opposite reaction, i.e. from $(+)-\alpha-\mathrm{HBCD}$ to $(+)-\gamma-\mathrm{HBCD}$, is much lower with $2.6 \cdot 10^{-3}$. Again, these results clearly confirm experimental observations concerning the increase of $\alpha$-HBCD and the decrease of $\gamma$-HBCD Peled et al. (1995). The reverse interconversion seems to be quite rare due to the unlikeliness of an anti conformation at the $\mathrm{C}_{1} \mathrm{Br}-\mathrm{C}_{2} \mathrm{Br}$-moiety of $\alpha-\mathrm{HBCD}$, presenting this stereoisomer as a thermodynamical sink of HBCD. 


\begin{tabular}{|c|c|c|c|c|c|c|}
\hline \multirow[t]{2}{*}{ HBCD diastereomer } & \multirow[t]{2}{*}{ Dihedral } & \multicolumn{2}{|c|}{ Global minimum } & \multirow[t]{2}{*}{$\frac{\mathrm{kJ}}{\mathrm{mol}}$} & \multirow[t]{2}{*}{ Boltzmann ratio } & \multirow[t]{2}{*}{ Target HBCD } \\
\hline & & & gauche & & & \\
\hline \multirow{3}{*}{$(+)-\alpha$} & $\mathrm{C}_{1} \mathrm{C}_{2}$ & 253.7 & 238.7 & & $2.6 \cdot 10^{-3}$ & $(+)-\gamma$ \\
\hline & $\mathrm{C}_{5} \mathrm{C}_{6}$ & 272.6 & 238.7 & & $1.3 \cdot 10^{-6}$ & $(+)-\beta$ \\
\hline & $\mathrm{C}_{9} \mathrm{C}_{10}$ & 272.6 & 238.7 & & $1.3 \cdot 10^{-6}$ & $(+)-\beta$ \\
\hline \multirow{3}{*}{$(+)-\beta$} & $\mathrm{C}_{1} \mathrm{C}_{2}$ & 263.6 & 249.5 & & $3.6 \cdot 10^{-3}$ & $(-)-\beta$ \\
\hline & $\mathrm{C}_{5} \mathrm{C}_{6}$ & 285.3 & 249.5 & & $6.1 \cdot 10^{-7}$ & $(-)-\gamma$ \\
\hline & $\mathrm{C}_{9} \mathrm{C}_{10}$ & 278.4 & 249.5 & & $9.5 \cdot 10^{-6}$ & $(+)-\alpha$ \\
\hline \multirow{3}{*}{$(+)-\gamma$} & $\mathrm{C}_{1} \mathrm{C}_{2}$ & 256.7 & 256.7 & & 1 & $(+)-\alpha$ \\
\hline & $\mathrm{C}_{5} \mathrm{C}_{6}$ & 275.1 & 256.7 & & $6.2 \cdot 10^{-4}$ & $(-)-\beta$ \\
\hline & $\mathrm{C}_{9} \mathrm{C}_{10}$ & 289.5 & 256.7 & & $2.0 \cdot 10^{-6}$ & $(-)-\beta$ \\
\hline \multirow{3}{*}{$(-)-\alpha$} & $\mathrm{C}_{1} \mathrm{C}_{2}$ & 253.7 & 238.7 & & $2.6 \cdot 10^{-3}$ & $(-)-\gamma$ \\
\hline & $\mathrm{C}_{5} \mathrm{C}_{6}$ & 272.6 & 238.7 & & $1.3 \cdot 10^{-6}$ & $(-)-\beta$ \\
\hline & $\mathrm{C}_{9} \mathrm{C}_{10}$ & 272.6 & 238.7 & & $1.3 \cdot 10^{-6}$ & $(-)-\beta$ \\
\hline \multirow{3}{*}{$(-)-\beta$} & $\mathrm{C}_{1} \mathrm{C}_{2}$ & 263.6 & 249.5 & & $3.6 \cdot 10^{-3}$ & $(+)-\beta$ \\
\hline & $\mathrm{C}_{5} \mathrm{C}_{6}$ & 285.3 & 249.5 & & $6.1 \cdot 10^{-7}$ & $(+)-\gamma$ \\
\hline & $\mathrm{C}_{9} \mathrm{C}_{10}$ & 278.4 & 249.5 & & $9.5 \cdot 10^{-6}$ & $(-)-\alpha$ \\
\hline \multirow{3}{*}{$(-)-\gamma$} & $\mathrm{C}_{1} \mathrm{C}_{2}$ & 256.7 & 256.7 & & 1 & $(-)-\alpha$ \\
\hline & $\mathrm{C}_{5} \mathrm{C}_{6}$ & 275.1 & 256.7 & & $6.2 \cdot 10^{-4}$ & $(+)-\beta$ \\
\hline & $\mathrm{C}_{9} \mathrm{C}_{10}$ & 289.5 & 256.7 & & $2.0 \cdot 10^{-6}$ & $(+)-\beta$ \\
\hline
\end{tabular}

Table 6. Global potential energy minima of both conformational subspaces, anti and gauche of each $\mathrm{C}_{i} \mathrm{Br}-\mathrm{C}_{i+1} \mathrm{Br}$-moiety of each $\mathrm{HBCD}$ diastereomer and respective Boltzmann ratios. The higher this value, the more likely the anti conformation and thus, the more likely the diastereomer to interconvert at the concerning moiety to the target diastereomer shown in the last column.

\subsection{Thermodynamical energy reweighting}

An estimation of interconversion rates of the main six HBCD diastereomers for the desired temperature $433 \mathrm{~K}$, i.e. with $\beta_{1}=0.2778 \mathrm{~mol} / \mathrm{kJ}$, required the computation of several thermodynamical quantities at the initial simulation temperature $1500 \mathrm{~K}\left(\beta_{0}=\right.$ $0.0802 \mathrm{~mol} / \mathrm{kJ})$. These were mean potential energies $\langle V\rangle\left(\beta_{0}\right)$ of each HBCD stereoisomer and differences of the mean potential energy $\Delta_{\text {ga }}\langle V\rangle\left(\beta_{0}\right)$, of the globally minimal potential energies $\Delta_{\mathrm{ga}} V^{0}$ and of free energies $\Delta_{\mathrm{ga}} A\left(\beta_{0}\right)$ associated with the dihedral angle of each $\mathrm{C}_{i} \mathrm{Br}-\mathrm{C}_{i+1} \mathrm{Br}$-moiety. Afterwards, all of these energies but the global minima at absolute zero were reweighted to the desired temperature.

Mean potential energies $\langle V\rangle(\beta)$ of the complete conformational space were easily derived from the high-temperature simulation data for each HBCD diastereomer in the order $(+)-\alpha$, $(+)-\beta,(+)-\gamma,(-)-\alpha,(-)-\beta$ and $(-)-\gamma: 920.6,930.9,926.3,922.0,931.2$ and $925.9 \mathrm{~kJ} / \mathrm{mol}$, respectively. The correponding mean potential energies associated with $433 \mathrm{~K}$ were obtained by applying the linear model of the reweighting formula (Equation 15) to the original mean potential energies resulting in 435.6, 446.2, 450.0, 436.0, 446.3 and $449.9 \mathrm{~kJ} / \mathrm{mol}$, respectively. Free $\left(\Delta_{\text {ga }} A\right)$ and mean potential $\left(\Delta_{\text {ga }}\langle V\rangle\right)$ energy differences associated with anti and gauche subspaces of each $\mathrm{C}_{i} \mathrm{Br}-\mathrm{C}_{i+1} \mathrm{Br}$-moiety and for both temperatures are presented in Table 7. Potential energy differences at absolute zero $\left(\Delta_{\mathrm{ga}} V^{0}\right)$ in the table were obtained by the difference of global potential energy minima of anti and gauche conformations at simulation temperature, since these are the conformations, towards which the molecular system moves with decreasing temperature. 


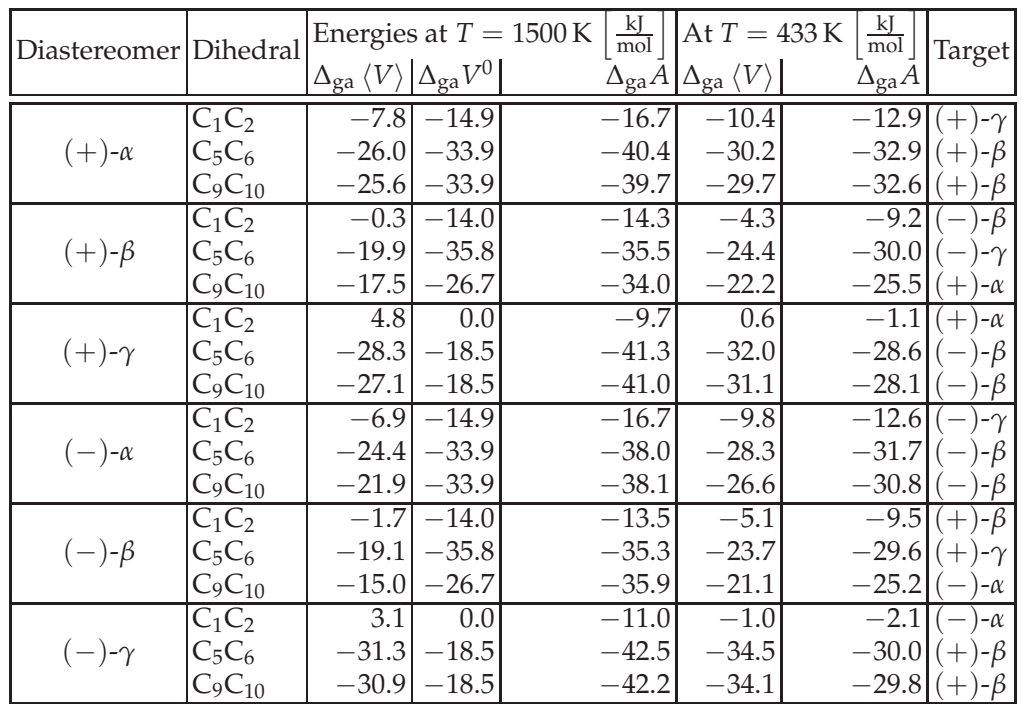

Table 7. Mean potential $\left(\Delta_{\text {ga }}\langle V\rangle\right)$ and free $\left(\Delta_{\text {ga }} A\right)$ energies of both conformational subspaces (anti and gauche) of each $\mathrm{C}_{i} \mathrm{Br}-\mathrm{C}_{i+1} \mathrm{Br}$-moiety of each HBCD diastereomer at the simulation temperature $T=1500 \mathrm{~K}$ and reweighted to the desired temperature at $T=433 \mathrm{~K}$, respectively as well as potential energy differences at $T=0 \mathrm{~K}$, i. e. potential energy differences of the concerning global minima $\left(\Delta_{\text {ga }} V^{0}\right)$ gained by sampling at $T=1500 \mathrm{~K}$ and minimizing.

The estimated equilibrium distribution for $433 \mathrm{~K}$ presented in section 6.3 is similar to the experimentally determined distribution after thermal rearrangement Peled et al. (1995). Both methods provide a by far highest fraction of $(+)-\alpha-\mathrm{HBCD}$ and decreasing fractions in the order $(+)-\beta$-HBCD and $(+)-\gamma$-HBCD. If the mean potential energies had been chosen to be interpolated to $630 \mathrm{~K}$ instead, the estimated equilibrium distribution would have resulted in equal values with a relative error of only $10 \%$, which is not presented among these results.

\subsection{HBCD interconversion rates and kinetics}

By inserting the negated free energy differences from the last but one column of Table 7 into the modified Arrhenius Equation 21, the embedded Markov chain was calculated first:

$$
K=\left(\begin{array}{cccccc}
0 & 0.0080 & 0.9920 & 0 & 0 & 0 \\
0.0107 & 0 & 0 & 0 & 0.9863 & 0.0031 \\
0.9990 & 0 & 0 & 0 & 0.0010 & 0 \\
0 & 0 & 0 & 0 & 0.0112 & 0.9888 \\
0 & 0.9838 & 0.0037 & 0.0126 & 0 & 0 \\
0 & 0.0009 & 0 & 0.9991 & 0 & 0
\end{array}\right) .
$$

This matrix contains a 0 whenever a interconversion is not possible. Only the 14 interconversions as depicted in Fig. 5 lead to values greater than 0 in matrix $K$. Row sums were scaled to 1 .

The steady-state distribution $\pi$ of $\mathrm{HBCD}$ at $433 \mathrm{~K}$ was determined by inserting the corresponding mean potential energy values, interpolated to this temperature as shown 
above, into the potential energy part of the Boltzmann expression, neglecting the partion function but scaling the sum to 1 :

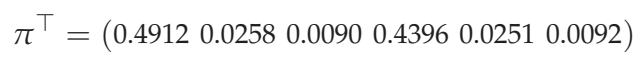

This vector gives evidence about a theoretical distribution of $\mathrm{HBCD}$ diastereomers. Summing up the first and forth value of $\pi$, i. e. the molar fractions of $(+)$ - and ( -$)-\alpha$-HBCD yields $93.1 \%$ for $\alpha$-HBCD and analogously, in case of $\beta$ - and $\gamma$-HBCD $5.1 \%$ and $1.8 \%$, respectively.

After having solved the eigenproblem of Equation 26, the theoretical rate matrix $Q$ was determined

$$
Q=\mu \cdot\left(\begin{array}{cccccc}
-0.0184 & 0.0001 & 0.0183 & 0 & 0 & 0 \\
0.0023 & -0.2200 & 0 & 0 & 0.2170 & 0.0007 \\
0.9990 & 0 & -1.0000 & 0 & 0.0010 & 0 \\
0 & 0 & 0 & -0.0167 & 0.0002 & 0.0166 \\
0 & 0.2231 & 0.0008 & 0.0028 & -0.2267 & 0 \\
0 & 0.0007 & 0 & 0.7879 & 0 & -0.7886
\end{array}\right)
$$

up to an unknown scaling factor $\mu$ which is due to the unknown velocity of the interconversion process. Consequently, simulation of the interconversion kinetics was performed now by applying Equation 27 to an initial distribution $x(0)$

$$
x^{\top}(0)=\left(\begin{array}{llllll}
0 & 0 & 1 & 0 & 0 & 0
\end{array}\right)
$$

with a discretized time step $\Delta \tau=1$ over a time span of $\tau=4000$ "seconds" and an arbitrary scaling factor $\mu=0.007$ resulting in interconversion kinetics as shown in Fig. 10. The initial distribution with $100 \%(+)-\gamma$-HBCD was chosen in accordance with the experimental setup which had led to the experimental kinetics depicted in Fig. 6. After these completely theoretical results of HBCD interconversion kinetics starting at $100 \%(+)-\gamma$-HBCD (Fig. 10) as also done in the laboratory, the concentration of this stereoisomer rapidly decreases with the highest rate reaching its equilibrium value already after 1000 "seconds", while the concentration of $(+)-\alpha$-HBCD increases with nearly the same velocity far above its equilibrium value. A longer simulation that is not presented here shows an subsequent decrease towards its equilibrium. The $\beta$ diastereomers are the next ones to increase towards their steady-state concentration with $(-)-\beta-\mathrm{HBCD}$ starting slightly faster due to its creation from $(+)-\gamma-\mathrm{HBCD}$. But with increasing $(+)-\alpha-\mathrm{HBCD},(+)-\beta-\mathrm{HBCD}$, which evolves from $(+)-\alpha-\mathrm{HBCD}$, accumulates faster and overtakes $(-)-\beta$-HBCD. The by far slowest growth is in the case of $(-)-\gamma$-HBCD.

From a qualitative point of view, all these observations on the kinetics correspond exactly with experimental results (Köppen et al. (2008)) as presented in Fig. 6 and Table 4. The highest and second highest rate was confirmed to be $k_{\gamma \rightarrow \alpha}$ and $k_{\beta \rightarrow \beta}$, respectively, whereas $k_{\alpha \rightarrow \beta}$ $(Q(1,2)$ and $Q(4,5))$ yielded the lowest value with both approaches. Many assumptions were necessary in order to estimate interconversion rates for the six main HBCD isomers, started off with the vacuum approximation, neglecting any intermolecular interactions in the liquid phase and by the way, reducing the computational effort. Due to sterical reasons and the fact of poor solubility in a polar medium such as water (Janak et al. (2005)), we claim a negligible affect of intermolecular interactions on the interconversion process.

Describing the quantum-chemically motivated inversion process in terms of classical mechanics was a second bold approximation. However, if we consider its rate as a combination of the probability of being activated (anti conformation) and the transition itself and under the assumption of an identical velocity of the latter part for all diastereomers, we 

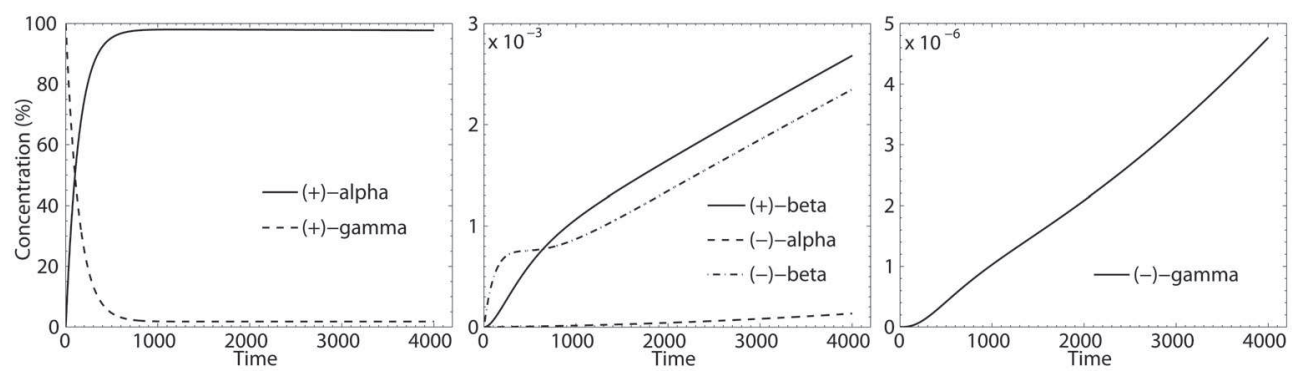

Fig. 10. theoretical determinantion of the HBCD isomerization at $160 \mathrm{C}$ starting at $100 \%$ $(+)-\gamma-\mathrm{HBCD}$ and with a scale factor $\mu=0.007$. Free and potential energy values had been reweighted after a high-temperature HMC simulation at $1500 \mathrm{~K}$.

are able to reduce the interconversion to a classical manner with its rate characterized by differences of energies/probabilities between anti and gauche subspaces, i.e. the difference in free energies expressing the likeliness of a molecular system to adopt the one or the other conformation. What we lose by this simplification is the knowledge about the absolute velocities of the interconversion and thus, the opportunity to describe rates quantitatively. But what we gain is the ability to calculate this quantum-chemical process (in reasonable time).

Also the application of the Arrhenius equation to these free energy differences might seem a little far-fetched, but again, there is an obvious correlation between the free energy difference and the ordinary activation energy. Both describe the delay of energies from an arbitrarily fixed value, differing in accordance with the direction of the reaction. In other words, if the activation energy is low for the forward direction, then the free energy difference is low as well. From the reverse perspective, the free energy difference increases, too, if the activation energy increases. Similar to the free energy difference, the activation energy may be considered in this context as the energy necessary to lead the molecules to the active ant $i$ conformation. Still, we are able to gain qualitative or even semi-quantitative results.

As we were interested in the kinetics of the HBCD system at $433 \mathrm{~K}$, a reweighting of energy values became unavoidable. Instead of a point-wise scheme that weights up a probably small number of values from the overlap region of high- and low-temperature distributions, we decided to apply a thermodynamical approach assuming a linear temperature-dependency of the mean potential energy (Equation 15). If the potential energy $V$ was a quadratic function in the onformational space $\Omega$, this assumption would be true. However, the potential energy function is not an exact quadratic one but is locally approximable by parabolic functions due to its Gaussian-like distributions in a accordingly decomposed conformational space.

In spite of all these approximations, the results well reflect the HBCD interconversion process as determined by experimental methods, and in particular, no experimental data was used for the estimation at all.

\section{References}

Akhmatskaya, E. \& Reich, S. (2004). The targeted shadowing hybrid Monte-Carlo (tshmc) method, Technical report, Institut für Mathematik, Uni Potsdam.

Allen, M. P. \& Tildesley, D. J. (1987). Computer Simulations of Liquids, Clarendon, Oxford.

Andersen, H. C. (1980). Molecular dynamics simulations at constant pressure and/or temperature, J.Chem.Phys. 72: 2384-2393. 
Barda, H. J., Sanders, D. C. \& Benya, T. J. (1985). Bromine compounds, in S. Hawkins \& G. Schultz (eds), Ullmann's Encyclopedia of Industrial Chemistry, Wiley-VCH, Weinheim, Germany, pp. 405-429.

Barontini, F., Cozzani, V. \& Petarca, L. (2003). Thermal stability and decomposition products of hexabromocyclododecane, Ind. Eng. Chem. Res. 40: 3270-3280.

Becher, G. (2005). The stereochemistry of 1,2,5,6,9,10-hexabromocyclododecane and its graphic representation, Chemosphere 58: 989-991.

Binder, K. \& Landau, D. (2000). A Guide to Monte Carlo Simulations in Statistical Physics, Cambirdge University Press, Cambirdge.

Clamp, M. E., Baker, P. G., Stirling, C. J. \& Brass, A. (1994). Hybrid Monte Carlo: An efficient algorithm for condensed matter simulation, J.Comput.Chem. 15(8): 838-846.

Covaci, A., Gerecke, A. S. C., Voorspoels, R. J. L. S., Kohler, M., Heeb, N. V., Leslie, H., Allchin, C. R. \& DeBoer, J. (2006). Hexabromocyclododecanes (HBCDs) in the environment and humans: a review, Environ. Sci. Techno. 40: 3680-3688.

de Wit, C. A., Alaee, M. \& Muir, D. C. G. (2006). Levels and trends of brominated flame retardants in the arctic, Chemosphere 64: 209-233.

de Witt, C. A. (2002). An overview of brominated flame retardants in the environment, Chemosphere 46: 583-624.

Deuflhard, P. (1983). Order and stepsize control in extrapolation methods, Numer. Math. pp. 399-422.

Deuflhard, P. (1985). Recent progress in extrapolation methods for ordinary differential equations, SIAM Review 27: 505-535.

Deuflhard, P., Dellnitz, M., Junge, O. \& Schütte, C. (1999). Computation of essential molecular dynamics by subdivision techniques, in P. D. et al (ed.), Computational Molecular Dynamics: Challenges, Methods, Ideas, Springer, Berlin, pp. 98-115.

Duane, S., Kennedy, A. D., Pendleton, B. J. \& Roweth, D. (1987). Hybrid Monte Carlo, Phys. Lett. B 195(2): 216-222.

Fischer, A. (1997). Die Hybride Monte-Carlo Methode in der Molekülphysik. Diploma thesis, Department of Mathematics and Computer Science, Free University Berlin, (in German).

Fischer, A., Cordes, F. \& Schütte, C. (1998). Hybrid Monte Carlo with adaptive temperature in a mixed-canonical ensemble: Efficient conformational analysis of RNA, J. Comput. Chem. 19: 1689-1697.

Forrest, B. M. \& Suter, U. W. (1994). Hybrid Monte Carlo simulations of dense polymer systems, J.Chem.Phys. 101.

Frenkel, D. \& Smit, B. (1996). Understanding molecular simulation. From algorithms to applications, Academic Press, San Diego.

Frenkel, D. \& Smit, B. (2002). Understanding Molecular Simulation - From Algorithms to Applications, Vol. 1 of Computational Science Series, 2nd edn, Academic Press, A Division of Harcourt, Inc., www.academicpress.com/computationalscience.

Gelman, A. \& Rubin, D. (1992). Inference from Iterative Simulation using Multiple Sequences, Statist. Sci. 7: 457-511.

Göpel, W. \& Wiemdörfer, H. D. (2000). Statistische Thermodynamik, Spektrum Akademischer Verlag.

Griebel, M., Zumbusch, G. \& Knapek, S. (2007). Numerical Simulation in Molecular Dynamics, Vol. 5 of Texts in Computational Science and Engineering, Springer, Berlin. 
Gromov, D. G. \& de Pablo, J. J. (1995). Structure of binary polymer blends - multiple time step hybrid Monte Carlo simulations and self-consistend intergal-equation theory, J.Chem.Phys. 103(18): 8247-8256.

Gupta, S., Irbäck, A., Karsch, F. \& Petersson, B. (1990). The acceptance probability in the hybrid Monte Carlo method, Phys. Lett. B 242: 437-443.

Hairer, E., Lubich, C. \& Wanner, G. (2004). Geometric Numerical Integration Structure-Preserving Algorithms for Ordinary Differential Equations, Vol. 31 of Springer Series in Computational Mathematics, corr. 2nd edn, Springer.

Hairer, E., Nørsett, S. P. \& Wanner, G. (1993). Solving Ordinary Differential Equations I, Nonstiff Problems, 2nd edn, Springer-Verlag, Berlin, Heidelberg.

Hale, R. C., La Guardia, M. J., Harvey, E., Gaylor, M. O., Matt, T. \& Mainor, T. M. (2006). Brominated flame retardant concentrations and trends in abiotic media, Chemosphere 64: 181-186.

Halgren, T. A. (1996). Merck Molecular Force Field: I-V, J. Comp. Chem. 17(5-6): 490-641.

Hamers, T., Kamstra, J. H., Sonneveld, E., Murk, A. J., Kester, M. H. A., Andersson, P. L., Legler, J. \& Brouwer, A. (2006). In Vitro Profiling of the Endocrine-Disrupting Potency of Brominated Flame Retardants, Toxicol. Sci. 92: 157-173.

Hammersley, J. \& Handscomb, D. (1964). Monte Carlo Methods, J. Wiley, New York.

Hansmann, U. H. E., Okamoto, Y. \& Eisenmenger, F. (1996). Molecular dynamics, Langevin and hybrid Monte Carlo simulations in a multicanonical ensemble, Chem.Phys.Lett. 259: 321-330.

Heeb, N. V., Schweizer, W. B., Kohler, M. \& Gerecke, A. C. (2004). 1,2,5,6,9,10-hexabromocyclododecane - a class of compounds with a complex stereochemistry, Book of abstracts, Toronto, Canada, pp. 337-340. The Third International Workshop on Brominated Flame Retardants.

Heeb, N. V., Schweizer, W. B., Mattrel, P., Haag, R., Kohler, M., Schmid, P., Zennegg, M. \& Wolfensberger, M. (2008). Regio- and stereoselective isomerization of hexabromocyclododecanes (HBCDs): Kinetics and mechanism of $\beta$-HBCD racemization, Chemosphere 71: 1547-1556.

Hestenes, M. R. \& Stiefel, E. (1952). Methods of Conjugate Gradients for Solving Linear Systems, J. Res. Nat. Inst. Stand. Technol. 49: 409-436.

Hockney, R. W. (1970). The potential calculations and some applications, Methods Comput. Phys. 9: 136-211.

Hunziker, R. W., Gonsior, S., MacGregor, J. A., Desjardins, D., Ariano, J. \& Friederich, U. (2004). Fate and effect of hexabromocyclododecane in the environment, Organohalogen Compd 66: 2300-2305.

Irbäck, A. (1994). Hybrid Monte Carlo simulation of polymer chains, J.Chem.Phys. 101(2): 1661-1667.

Izaguirre, J. A. \& Hampton, S. S. (2004). Shadow hybrid Monte Carlo: An efficient propagator in phase space of macromolecules, J. Comput. Phys. 200: 581-604.

Janak, K., Covaci, A., Voorspoels, S. \& Becher, G. (2005). Hexabromocyclododecane in marine species from the western Scheldt estuary: diastereoisomer- and enantiomer-specific accumulation, Environ. Sci. Technol. 39: 1987-1994.

Johnson-Restrepo, B., Adams, D. H. \& Kannan, K. (2008). Tetrabromobisphenol A (TBBPA) and hexabromocyclododecanes (HBCDs) in tissues of humans, dolphins, and sharks from the United States, Chemosphere 70(11): 935-1944. 
Kennedy, A. D. \& Pendleton, B. (1991). Acceptances and autocorrelation in hybrid Monte Carlo, Nuclear Physics B Proceedings Supplements 20: 118-121.

Kijima, M. (1997). Markov Processes for Stochastic Modeling, Stochastic Modeling Series, Chapman and Hall.

Köppen, R., Becker, R., Jung, C. \& Nehls, I. (2008). On the thermally induced isomerisation of hexabromocyclododecane stereoisomers, Chemosphere 71: 656-662.

Law, K., Palace, V. P., Halldorson, T., Danell, R., Wautier, K., Evans, B., Alaee, M., Marvin, C. \& Tomy, G. T. (2006a). Dietary accumulation of hexabromocyclododecane diastereoisomers in juvenile rainbow trout (Oncorhynchus mykiss) I: Bioaccumulation parameters and evidence of bioisomerization, Environ. Toxicol. Chem. 25: 1757-1761.

Leach, A. (2001). Molecular Modelling - Principles and Applications, Pearson Educations Ltd., Essex.

Leimkuhler, B. \& Reich, S. (2004). Simulating Hamiltonian Dynamics, Cambridge.

Lew, A., Marsden, J. E., Ortiz, M. \& West, M. (2004). An overview of variational integrators, in L. P. Franca, T. E. Tezduyar \& A. Masud (eds), Finite Element Methods: 1970's and Beyond, CIMNE, ISBN 84-95999-49-8, pp. 98-115.

Manousiouthakis, V. I. \& Deem, M. W. (1999). Strict detailed balance is unnecessary in Monte Carlo simulation, J. Chem. Phys. 110: 2753-2756.

Meerts, I. A. T. M., van Zanden, J. J., Luijks, E. A. C., van Leeuwen-Bol, I., Marsh, G., Jakobsson, E., Bergman, A. \& Brouwer, A. (2000). Potent Competitive Interactions of Some Brominated Flame Retardants and Related Compounds with Human Transthyretin in Vitro, Toxicol. Sci. 56: 95-104.

Mehlig, B., Heermann, D. W. \& Forrest, B. M. (1992). Hybrid Monte Carlo method for condensed-matter systems, Phys.Rev. B 45(2): 679-685.

Metropolis, N., Rosenbluth, A. W., Rosenbluth, M. N., Teller, A. N. \& Teller, E. (1953). Equation of state calculations by fast computing machines, J.Chem.Phys. 21: 1087-1092.

Peled, M., Scharia, R. \& Sondack, D. (1995). Thermal rearrangement of hexabromocyclododecane (HBCD), in J. R. Desmurs, B. Gérard \& M. J. Goldstein (eds), Advances in Organobromine Chemistry II, Elsevier, Amsterdam, The Netherlands, pp. 92-99.

Peskun, P. (1973). Optimum Monte Carlo sampling using Marcov chains, Biometrika 60: 607-612.

Robert, C. P. \& Casella, G. (1999). Monte Carlo Statistical Methods, Springer New York, Berlin, Heidelberg.

Schäfer, K. (1960). Statistische Theorie der Materie, Vol. 1, Vandenhoeck \& Ruprecht in Göttingen. In German.

Schlick, T. (2002). Molecular Modelling and Simulation, Springer, New York.

Schriks, M., Zvinavashe, E., Furlow, J. D. \& Murk, A. J. (2006). Disruption of thyroid hormone-mediated Xenopus laevis tadpole tail tip regression by hexabromocyclododecane (HBCD) and 2,2' $, 3,3^{\prime}, 4,4^{\prime}, 5,5^{\prime}, 6$-nona brominated diphenyl ether (BDE206), Chemosphere 65: 1904-1908.

Skeel, R. D. \& Hardy, D. J. (2002). Practical construction of modified Hamiltonians, SIAM Journal on Scienti c Computing 23(4): 1172-1188.

Tomy, G. T., Budakowski, W., Halldorson, T., Whittle, D. M., Keir, M. J., Marvin, C., Macinnis, G. \& Alaee, M. (2004). Biomagnification of $\alpha$ - and $\gamma$-hexabromocyclododecane isomers in a Lake Ontario food web, Environ. Sci. Technol. 38: 2298-2303. 
Verlet, L. (1967). Computer "experiments" on classical fluids i. thermodynamical properties of lennard jones molecules, Phys. Rev. Vol. 159(No. 1): 98-103.

Vos, J. G., Becher, G., van den Berg, M., de Boer, J. \& Leonards, P. E. G. (2003). Brominated flame retardants and endocrine disruption, Pure Appl. Chem. 75: 2039-2046.

Weber, M. (2007). Conformation-Based Transition State Theory, Technical Report 07-14, Konrad-Zuse-Zentrum für Informationstechnik Berlin.

Wendlandt, J. M. \& Marsden, J. E. (1997). Mechanical integrators derived from a discrete variational principle, Physica D 106: 223-246.

Zhang, H. (1999). A new hybrid Monte Carlo algorithm for protein potential function test and structure refinement, Proteins 34: 464-471. 


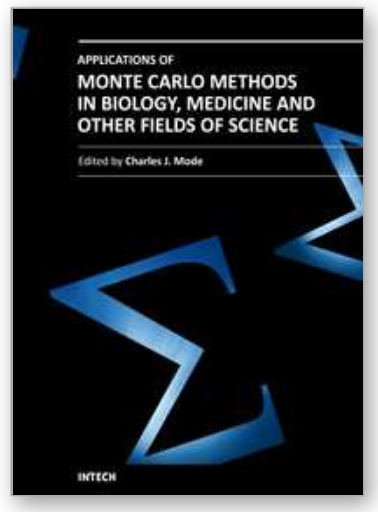

\section{Applications of Monte Carlo Methods in Biology, Medicine and Other Fields of Science}

Edited by Prof. Charles J. Mode

ISBN 978-953-307-427-6

Hard cover, 424 pages

Publisher InTech

Published online 28, February, 2011

Published in print edition February, 2011

This volume is an eclectic mix of applications of Monte Carlo methods in many fields of research should not be surprising, because of the ubiquitous use of these methods in many fields of human endeavor. In an attempt to focus attention on a manageable set of applications, the main thrust of this book is to emphasize applications of Monte Carlo simulation methods in biology and medicine.

\section{How to reference}

In order to correctly reference this scholarly work, feel free to copy and paste the following:

V. Durmaz, K. Fackeldey and M. Weber (2011). A rapidly Mixing Monte Carlo Method for the Simulation of Slow Molecular Processes, Applications of Monte Carlo Methods in Biology, Medicine and Other Fields of Science, Prof. Charles J. Mode (Ed.), ISBN: 978-953-307-427-6, InTech, Available from:

http://www.intechopen.com/books/applications-of-monte-carlo-methods-in-biology-medicine-and-other-fieldsof-science/a-rapidly-mixing-monte-carlo-method-for-the-simulation-of-slow-molecular-processes

\section{INTECH}

open science | open minds

\author{
InTech Europe \\ University Campus STeP Ri \\ Slavka Krautzeka 83/A \\ 51000 Rijeka, Croatia \\ Phone: +385 (51) 770447 \\ Fax: +385 (51) 686166 \\ www.intechopen.com
}

\author{
InTech China \\ Unit 405, Office Block, Hotel Equatorial Shanghai \\ No.65, Yan An Road (West), Shanghai, 200040, China \\ 中国上海市延安西路65号上海国际贵都大饭店办公楼 405 单元 \\ Phone: +86-21-62489820 \\ Fax: $+86-21-62489821$
}


(C) 2011 The Author(s). Licensee IntechOpen. This chapter is distributed under the terms of the Creative Commons Attribution-NonCommercialShareAlike-3.0 License, which permits use, distribution and reproduction for non-commercial purposes, provided the original is properly cited and derivative works building on this content are distributed under the same license. 